\title{
Stable Disarrangement Phases Arising from Expansion/Contraction or from Simple Shearing of a Model Granular Medium
}

\author{
L. Deseri ${ }^{a, c, d} \quad$ D. R. Owen ${ }^{b}$
}

March 31, 2015

\section{Abstract}

1

\section{Acknowledgements}

L. Deseri gratefully acknowledges financial support from the grant PIAPP-

GA-2013-609758-HOTBRICKS, "Mechanics of refractory materials at high temperature for advanced industrial technologies", from the EU through the FP7 program, as well as the hospitality of the Departments of Mathematical Sciences, Civil and Environmental Engineering, and Mechanical Engineering of Carnegie Mellon University.

The authors acknowledge the Center for Nonlinear Analysis at Carnegie Mellon University through the NSF Grant No. DMS-0635983.

\footnotetext{
${ }^{1 a}$ Dept. of Civil, Environmental and Mechanical Engineering, University of Trento, Via Mesiano 77, I-38123 Trento, Italy

${ }^{b}$ Center for Nonlinear Analysis and Department of Mathematical Sciences, Carnegie Mellon University, 4811 Frew St., Pittsburgh PA 15213-3890 USA

c Full affiliate member TMHRI-Department of Nanomedicine, The Methodist Hospital Research Institute, 6565 Fannin St., MS B-490 Houston, TX 77030 USA

${ }^{d}$ Dept. of Civil and Environmental Engineering and Dept. of Mechanical Engineering-CIT Carnegie Mellon University, Pittsburgh PA 15213-3890 USA

phone: +39 0461282511

deseri@andrew.cmu.edu
} 
Pietro Pollaci and Valentina Piccolo, grad. students of DICAM-University of Trento, are gratefully acknowledged for editing the figures. 


\title{
Stable Disarrangement Phases Arising from Expansion/Contraction or from Simple Shearing of a Model Granular Medium
}

July 24, 2015

\begin{abstract}
A principal challenge in modelling granular media is to connect the macroscopic deformation of the aggregate of grains with the average deformation of a small number of individual grains. We used in previous research the two-scale geometry of structured deformations $(g, G)$ and the theory of elastic bodies undergoing disarrangements (non-smooth submacroscopic geometrical changes) and dissipation to obtain, in terms of the free-energy response $\Psi$ of the body, an algebraic tensorial consistency relation between the macroscopic deformation $F=\nabla g$ and the grain deformation $G$, as well as an accommodation inequality $\operatorname{det} F \geq \operatorname{det} G>0$ that guarantees that the aggregate provides enough room at each point for the deformation of the grains. These two relations determine all of the disarrangement phases $G$ corresponding to a given $F$. We use the term stable disarrangement phase to denote a grain deformation $G$ that minimizes the stored energy density for the aggregate $\Psi\left(G^{\prime}\right)$ among all the disarrangement phases $G^{\prime}$ corresponding to $F$. Stability in this sense is determined solely by the constitutive response function $\Psi$ and, therefore, may be described as a notion of material stability with respect to changes in microstructure. In this article we determine for a model aggregate and
\end{abstract}


for two familiar families of macroscopic deformation - simple shears and uniform expansions or contractions - all of the stable as well as all of the unstable disarrangement phases of the model aggregate. For the stable disarrangement phases, we determine the connections between aggregate deformation and grain deformation. We showed in an earlier article that each stable disarrangement phase of this model aggregate cannot support tensile tractions, and our present results confirm that no-tension property of stable disarrangement phases for the model granular medium. Consequently, the appearance of tensile tractions in the present model granular medium would entail the loss of the material stability that we consider. This loss of stability is expected to be the rule, rather than the exception, because only special boundary conditions turn out to be compatible with one or more of the stable disarrangement phases at the disposal of the material.

\section{Introduction}

Principal challenges in modelling multiscale phenomena in continua are the description of the coupling between macroscopically observed geometrical changes and submacroscopically occurring geometrical changes, as well as the manner in which geometrical changes at different levels account for the internal dissipation arising during motions of the continuum. These challenges both were addressed [2] in a theory of elastic bodies capable both of undergoing disarrangements (non-smooth submacroscopic geometrical changes) as well as experiencing internal dissipation through temporally changing disar- 
rangements. In fact, the richer geometrical setting provided in [2] permits one to identify more precisely and physically the sources of internal dissipation during such temporal changes. In this article we restrict our attention to statical phenomena, so that the issue of internal dissipation is not considered explicitly here. (The interested reader may consult $[[2],[3],[7]]$ for detailed discussions of the issue of internal dissipation. In particular, the notion of material stability that we adopt here arises out of the extensive discussion of internal disspitation and submacroscopic stability of equilibrium configurations in [3].)

Thus, we here study in the setting of the multiscale geometry of structured deformations [1] the manner in which the macroscopic deformation of an aggregate of small elastic bodies that constitute a granular medium can be related to the submacroscopic deformation of the pieces of the aggregate. (We previously have used the terms "elastic aggregate" and "granular medium" synonymously, where the attribute "elastic" applies to the small pieces that form the aggregate, and not necessarily to the aggregate itself. The term "aggregate of elastic bodies", although longer than the term "elastic aggregate", is more precise and avoids the erroneous inference that the continuum, as a whole, is an elastic body in the classical sense. Henceforth, we use the term "aggregate of elastic bodies".)

Structured deformations provide an appropriate setting for studying aggregates of elastic bodies, because they entail purely geometrical fields 
$g$ and $G$ that distinguish between the macroscopic deformation of a continuum and the smooth geometrical changes that occur at submacroscopic length scales. In the case of an aggregate of elastic bodies, we may think of the point mapping $g$ as providing the macroscopic geometrical changes of the aggregate, as a whole, and we may think of the tensor field $G$ as providing a measure of the average geometrical changes of individual pieces (or grains) of the aggregate. The theory of structured deformations then justifies calling the field $M=\nabla g-G$ the deformation due to disarrangements, i.e., due to submacroscopic slips and separations among the pieces of the aggregate. Because we are interested here in a notion of material stability, we emphasize in this paper the case in which the aggregate undergoes a given, homogeneous deformation $g$ with gradient $\nabla g=F=$ const. while all of the pieces of the aggregate undergo a sequence of piecewise homogeneous deformations whose gradients, when averaged over small subbodies, converge to the constant tensor field G. Such emphasis is natural in studies of material properties such as material stability, as long as the response of the body at a point depend only upon piecewise affine approximations to geometrical changes at that point. The present study conforms to these requirements. Moreover, this emphasis arises also in classical studies of solid, liquid, and vapor phases of matter, in which the identification of the phases available to a body and analysis of their energetic properties can be carried out in the context of homogeneously deformed and heated bodies. (See [3] for a discussion in which broader classes of fields $g$ and $G$ are considered.) An attempt to model inhomogeneous deformations of grains within the theory of structured de- 
formations would require the use of "second-order structured deformations" [24] in which piecewise quadratic approximations underlie the kinematical changes at the microlevel. A corresponding theory of elastic bodies undergoing disarrangements and dissipation in the context of second-order structured deformations awaits development at this time.

The theory [2] of elastic bodies undergoing disarrangements and dissipation provides the consistency relation

$$
D_{G} \Psi(G, M)\left(F^{T}-G^{T}\right)+D_{M} \Psi(G, M) F^{T}=0,
$$

a tensorial relation involving the fields $F=\nabla g, G, M=F-G$ as well as the partial derivatives $D_{G} \Psi$ and $D_{M} \Psi$ of the Helmholtz free energy response $\Psi$ of the body. The consistency relation arises (see [20, 21], as well as [23] for details) because the stress $S$ in the reference configuration has both an additive decomposition $(\operatorname{det} K) S=S \backslash+S_{d}$, with $K=F^{-1} G$, and a multiplicative decomposition $(\operatorname{det} K) S=S \backslash K^{T}$. The stress without disarrangements $S \backslash$ and the stress due to disarrangements $S_{d}$ are defined by

$$
S \backslash=(\operatorname{det} K) S K^{-T}, \quad S_{d}=(\operatorname{det} K) S-S \backslash .
$$

These defining relations are universal in that they are meaningful for any continuum undergoing structured deformations, independent of the material comprising the body. The particular constitutive assumptions, $S_{\backslash}=$ $(\operatorname{det} K) D_{G} \Psi$ and $S_{d}=(\operatorname{det} K) D_{M} \Psi$, made in [2], permit us to interpret physically these refined measures of stress as driving 
forces associated with $G$ and $M$ respectively. These assumptions lead immediately to the stress relation

$$
S=D_{G} \Psi+D_{M} \Psi
$$

that determines the stress in the reference configuration as a function of $G$ and $M$.

In classical, finite elasticity, only structured deformations with $G=F$, i.e., only classical deformations are considered and, therefore, no disarrangements may occur during motions an elastic body in that setting, so that $M=0$ throughout the body, and no internal dissipation occurs. The theory [2] includes finite elasticity as a special case, enlarges the type of material response available to the body by admitting all pairs of fields $G$ and $M=F-G$ that satisfy the consistency relation, and allows the body to dissipate energy in smooth dynamical processes.

The theory [2] follows [1] in providing also the accommodation inequality, $0<\operatorname{det} G \leq \operatorname{det} F$, that guarantees that the macroscopic deformation $F$ provides enough volume to accommodate the submacroscopic geometrical changes associated with $G$. In the case of an arbitrary body undergoing structured deformations, the accommodation inequality guarantees that there are injective, piecewise affine approximations to the macroscopic deformation $g$ that converge in an appropriate sense to $g$ and whose gradients converge to $G$. In the case of an aggregate of elastic bodies, we interpret these approximations as deformations of the collection of individual grains that 


\section{do not cause interpenetration of pairs of grains.}

Together the consistency relation and accommodation inequality determine which tensors $G$ are compatible with a given macroscopic deformation gradient $F$. In $[3,20,21]$ we restricted attention to the case of purely dissipative disarrangements for which the free energy response $\Psi$ does not depend upon the disarrangement tensor $M$, so that no energy is stored via submacroscopic slips and separations, alone; in this case the consistency relation reduces to $D \Psi(G)\left(F^{T}-G^{T}\right)=0$. We defined in $[20$, 21] a disarrangement phase corresponding to $F$ to be a tensor $G$ that satisfies both the consistency relation and the accommodation inequality for the given $F$. Examples that we give in this paper and others given elsewhere $[2,3,20,21]$ show that typically there are multiple disarrangement phases corresponding to a given macroscopic deformation gradient $F$. Among them are the compact phase in which $G=F$ and, therefore, all of the grains deform in the same manner as the aggregate, itself, as well as a loose, stress-free phase $G=k R$, in which $R$ is a rotation and $k$ is a material constant (the accommodation inequality in the case of the loose phase requires that $k^{3} \leq \operatorname{det} F$.)

Our study of submacroscopic stability of equilibrium configurations in [3] employs a dissipation inequality for quasi-static evolutions of a body. The analysis there shows that the search for submacroscopically stable equilibrium configurations of a body undergoing disarrangements and dissipation leads directly to a non-increasing "augmented energy functional" and, subsequently, to the notion of material stability that we formulated in [20,21] 
and that we employ here: a stable disarrangement phase corresponding to $F$ is a disarrangement phase $G$ corresponding to $F$ that minimizes the Helmholtz free energy among all disarrangement phases corresponding to $F$. As indicated in [3], these stable disarrangement phases are candidates for limiting states toward which an isolated body evolves in the presence of dissipation. Thus, identification of stable disarrangement phases would provide a target landscape for the long-term evolution of an isolated aggregate of elastic bodies. For evolution without isolation, it is expected that coexistence of stable and unstable disarrangement phases would be the rule.

In the context of a familiar two-parameter class of free energy responses $\Psi_{\alpha \beta}$, our specific goals in this paper are:

- to determine all of the stable disarrangement phases corresponding to homogeneous expansions/contractions,

- to determine all of the stable disarrangement phases corresponding to simple shears,

- to describe in each of the two bullets above the relationship between the macroscopic deformation $F=\nabla g$ of the aggregate and the deformation $G$ of the pieces of the aggregate.

We illustrate now by means of two idealized, one-dimensional, isothermal stress-extension curves the idea of a disarrangement phase and compare it with the familiar notion of "phase of an elastic body". For the left-hand curve in Figure 1, the stress $T$ is a (single-valued) function of the extension $e$ with 

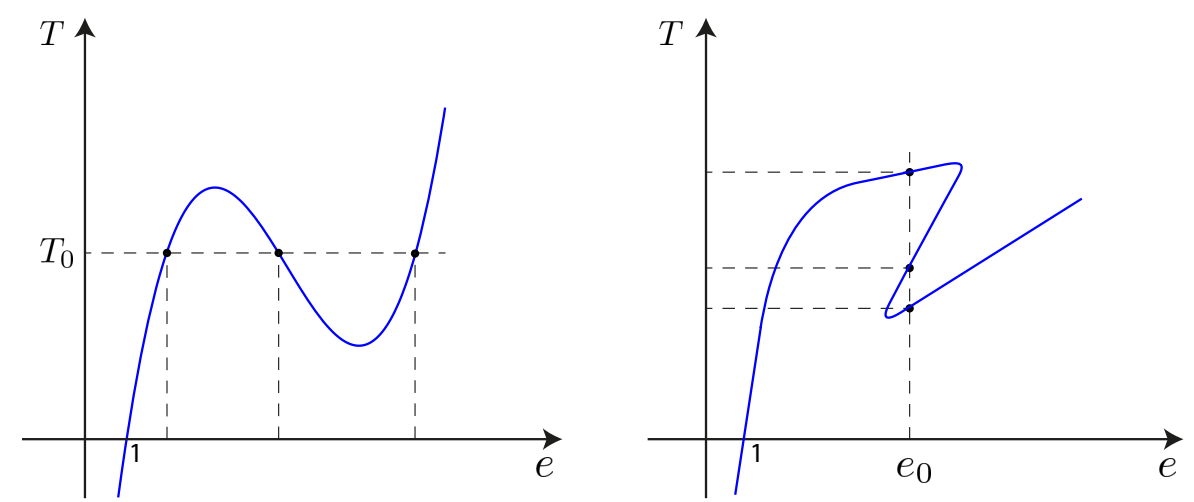

Figure 1: Standard phases and disarrangement phases.

the property that, for some values of stress, there are more than one value of extension that produce that stress. For example, the stress $T_{0}$ can be achieved at three different values of the extension, and it is customary to refer to the three values of $e$ corresponding to $T_{0}$ as phases of the elastic continuum corresponding to the stress $T_{0}$. In this standard notion of phase, different phases may be distinguished by differences in the macroscopic deformation of the body, and an important goal in the study of phases for continua is that of providing contexts in which coexistent phases, even fine mixtures of such phases, can be described and simulated.

By contrast, the stress-extension curve on the right of the figure does not provide a single stress value $T$ for each extension $e$, and we may fix the extension at the value $e_{0}$ and consider the three values of stress compatible with $e_{0}$ as corresponding to distinct phases of the material. Clearly, the macroscopic extension of the body cannot be used to distinguish among these phases, and it is natural to explore the possibility that disarrangements, i.e., non-smooth geometrical changes at submacroscopic length scales, may be used to distinguish among these phases. For both notions of "phase" illustrated here, one typically encounters a multiplicity of possible phases available to a given material, and an 
appropriate selection principle in the form of a stability criterion is required in order to narrow the list of competing phases. Our notion of stable disarrangement phase embodies this idea, is in accord with a notion of "submacroscopically stable equilibria of elastic bodies" introduced in [3], and is studied here in a fully three dimensional context for aggregates of elastic bodies.

In Section 2 we review the aspects of structured deformations and of the field theory [2] required for the present study. The field relations, including the consistency relation and the accommodation inequality, are given at the beginning of Section 2, and in Remark 1 we use the consistency relation to provide an inequality relating the energy when $G=F$ to that when $G$ differs from $F$ by a rank-one tensor. In Section 2.1 we recall from $[20,21]$ the definition of "disarrangement phase corresponding to $F "$ as well as the notion of "compact phase" (disarrangement-free phase) and, for a slightly less general class of aggregates, the notion of "loose phases" (stress-free phases) of an aggregate. In the compact phase, the disarrangement tensor $M$ vanishes, so that the pieces of the aggregate deform precisely as the aggregate through the macroscopic deformation gradient, i.e., $G=F=\nabla g$. In the loose phases, the pieces of the aggregate achieve a stress-free, energy minimizing state of deformation in which $G$ is a scalar times an arbitrary rotation tensor. The accommodation inequality shows that loose phases can only be present when the volume change $\operatorname{det} F$ of the macroscopic deformation is sufficiently large.

The notion of a "stable disarrangement phase" as an energy-minimizing disarrangement phase $[20,21]$ is reviewed in Section 2.2, and the notions of compact phase and loose phases are reexamined in light of this notion of material stability.

Section 2.3 provides a review from earlier studies [20], [7], [3] of the twoparameter class of free energy response functions $\Psi_{\alpha \beta}$ ( with $\alpha$ and $\beta$ "elastic 
constants" ) widely studied in the literature (see, for example, [19], Section 4.10). In Section 2.4 we record, for a given but arbitrary deformation gradient $F$, the complete catalog obtained in $[20,21]$ of solutions of the consistency relation when $\Psi=\Psi_{\alpha \beta}$. These solutions $G$ naturally form four categories: "compact," "plane-stress," "uniaxial stress." and "stress-free," the last three categories are identified according to the nature of the stress response $S$ that is calculated for each category of solutions. Because the consistency relation can be written in the tensorial form $S M^{T}=0$, the disarrangement tensors $M=F-G$ for these categories turn out to have ranks 0 , at most 1 , at most 2 , and at most 3 , respectively.

In Section 2.5 we describe a procedure for finding the stable disarrangement phases corresponding to an arbitrary macroscopic deformation gradient $F$. For the free energy response $\Psi_{\alpha \beta}$, we carry out in Sections 3 this procedure in detail for the one parameter family $F=\lambda^{1 / 2} I$ of uniform expansions/contractions, and in Section 4 for the one-parameter family $F=I+\mu a \otimes b$ of simple shears. We obtain a complete list in Section 3 of the stable disarrangement phases corresponding to $F=\lambda^{1 / 2} I$, and in Section 4.5 a complete list of the stable disarrangement phases corresponding to $F=I+\mu a \otimes b$, conveniently described in terms of the maximum principal stretch $\lambda:=1+\left(\mu^{2}+|\mu|\left(\mu^{2}+4\right)^{1 / 2}\right) / 2>1$ for $\mu \neq 0$. The availability of these lists in the cases of expansion or contraction and in the case of simple shear provides detailed and specific insights into the dependence of $G$ upon $F$ when $G$ is a stable disarrangement phase, and provides in Figures 2 and 5 partitions of the $\lambda-r$ plane into regions in each of which only one stable disarrangement phase (or, perhaps, only one class of equivalent phases) may arise. Figures 2 and 5 are counterparts of phase diagrams familiar in the study of standard phases of elastic bodies. For the stable disarrangement phases identified in Section 4.5 we are able to determine in Section 4.6 detailed 
connections between, on the one hand, the principal stretches and directions for $F$ and, on the other, the principal stretches and directions for $G$. (The stable disarrangement phases found in Section 3 consist only of the compact phase and the loose phase for which the nature of $G$ and its relationship to $F$ were provided already in Section 2.1.)

The explicit results that we obtain here show that, for the two classes of macroscopic deformation gradients $F$ studied in Sections 3 and 4, the stable disarrangement phases corresponding to $F$ never result in tensile tractions within the body. These conclusions confirm the result established in [20, 21] for arbitrary $F$ : stable disarrangement phases associated with $\Psi_{\alpha \beta}$ cannot support tensile tractions. This property comes into play in Sections 3 and 4 where, for each given value of the ratio $r=\alpha / \beta$, there is a particular value $\hat{\lambda}(r)$ of the stretch parameter $\lambda$ at which one of the principal stresses arising for the compact phase passes from negative values through zero to positive values as $\lambda$ increases through $\hat{\lambda}(r)$, and stability of the compact phase thereby is lost. This loss of stability of the compact phase occurs in spite of the fact that the free energy $\Psi_{\alpha \beta}$ is smooth and rank-one convex. In particular, $\Psi_{\alpha \beta}$ satisfies the Legendre-Hadamard condition and, hence, does not admit material instabilities associated with loss of ellipticity (see e.g. [13, 14, 16, 17, 18]).

We note that, for the stable plane-stress phase corresponding to the simple shear $F=I+\mu a \otimes b$, the free energy depends only upon the elastic constants $\alpha$ and $\beta$ and not upon the amount of macroshear $\mu$. This fluid-like behavior stands in contrast to the solid-like dependence of the free energy upon $\mu$ for the compact phase, and this result is in agreement with the observed ability of aggregates to exhibit both solid-like and fluid-like behavior [7], [8], [9], [10].

Our detailed results also include information about disarrangement phases that lose the competition for the status of minima of the energy. Although 
energetically less favorable than their stable competitors, these unstable but in some cases energetically stationary disarrangement phases will play a role in the solution of boundary-value problems in the statics of aggregates of elastic bodies $\backslash$. In particular, boundary tractions computed from stresses arising in stable disarrangement phases may not agree with prescribed boundary tractions, as is the case for the loose phase in the presence of non-zero prescribed boundary tractions, thereby necessitating the formation of zones of unstable disarrangement phases near the boundary. The likely appearance of unstable disarrangement phases in solutions of boundaryvalue problems foreshadows the possibility that tensile tractions may also appear. Moreover, while we have not modelled aggregates of elastic bodies as bodies with internal friction, the appearance in our approach of boundaries separating adjacent regions occupied by different disarrangement phases [7] would allow one to determine whether or not a particular frictional response is present and, hence, accountable for the emergence of coexisting phases.

\section{Summary of concepts and results from earlier studies}

The multiscale geometry provided by structured deformations [1] has been applied [7] to describe moving phase interfaces in a granular medium composed of small elastic bodies that can deform individually in a manner that differs from the macroscopic deformation of the continuum. In $[20,21]$ we specialized that description to a body that does not evolve in time, so that geometrical changes of 
the granular medium may be described by structured deformations $(g, G)$. Each such structured deformation provides the macroscopic deformation $g: \mathcal{B} \longrightarrow \mathcal{E}$ mapping points $X$ in the body $\mathcal{B}$ injectively into Euclidean space $\mathcal{E}$ as well as the deformation without disarrangements $G: \mathcal{B} \longrightarrow$ Lin mapping points $X$ in the body into second-order tensors $G(X)$ that describe the deformation of pieces of the granular medium. The definition of structured deformation includes the requirement that the fields $g$ and $G$ satisfy the accommodation inequality [1] at each point $X$ in the body:

$$
0<m<\operatorname{det} G(X) \leq \operatorname{det} \nabla g(X)
$$

Here, $m$ is a positive number that does not depend upon $X, \nabla g$ is the classical derivative of the macroscopic deformation, and det denotes the determinant. This inequality reflects the idea that the macroscopic deformation should provide enough room to accommodate all of the pieces of the aggregate without causing interpenetration of matter.

The ability of the pieces of the aggregate to deform differently from the aggregate, itself, gives rise to slips and separations among the individual piecescalled disarrangements. The accomodation inequality (1) can be used to prove the Approximation Theorem [1]: there exists a sequence $n \longmapsto f_{n}$ of injective, piecewise-smooth mappings of the body into Euclidean space such that

$$
g=\lim _{n \longrightarrow \infty} f_{n} \text { and } G=\lim _{n \longrightarrow \infty} \nabla f_{n}
$$

where for present purposes the sense of convergence in the two limits need not be made explicit. Thus $G$, as a limit of classical derivatives, reflects at the macrolevel the smooth deformation away from any submacroscopic sites of disarrangements associated with the piecewise smooth approximates $f_{n}$. In 
addition, it has been shown [1], [11] that the tensor field

$$
M=\nabla g-G
$$

captures the average of the submacroscopic separations and slips embodied in the jumps of the approximates $f_{n}$, and we are justified in calling $M$ the deformation due to disarrangements. The piecewise smooth approximations $f_{n}$ may be viewed as snapshots of the deforming aggregate taken with magnification sufficient to reveal the individual pieces of the aggregate.

We note that general elastic bodies undergoing disarrangements can store energy through both the deformation without disarrangements $G$ and the deformation due to disarrangements $M$ [2]. In order to specialize to the situation in which the slips and separations between pieces of the aggregate are purely dissipative, i.e., do not themselves contribute to the stored energy, it was assumed in [7] that the Helmholtz free energy field $\psi$ for the aggregate is determined entirely by the deformation without disarrangements $G$, which at each point $X$ in the reference configuration amounts to the relation:

$$
\psi(X)=\Psi(G(X))
$$

where $\Psi$ is a smooth constitutive function, and $\psi(X)$ is the free energy per unit volume in the reference configuration. The constitutive equation (4) for an aggregate undergoing purely dissipative disarrangements can be derived from the assumption that (i) the energy associated with the piecewise smooth approximations $f_{n}$ has no interfacial term and that (ii) the convergence in (2) is essentially uniform and $\Psi$ is continuous. (See [12], Part Two, Section 2 for the supporting mathematical reasoning). This amounts to assuming that each piece of the aggregate is an elastic body with energy density response $\Psi$ and 
that no energy is stored when pieces of the aggregate rotate, separate, or slide relative to one another.

The general field equations for elastic bodies undergoing disarrangements and dissipation [2] reduce in the present, statical context and in the presence of purely dissipative disarrangements to the system

$$
\begin{gathered}
\operatorname{div} D \Psi(G)+b=0 \\
D \Psi(G)(\nabla g-G)^{T}=0 \\
0<\operatorname{det} G \leq \operatorname{det} \nabla g
\end{gathered}
$$

in which (5) is the equation of balance of forces, (6) is a tensorial equation called the consistency relation that reflects the fact the the stress tensor in a continuum undergoing disarrangements has both an additive and a multiplicative decomposition (see the appendix in Part II of [20] and [2] for details), and (7) is a weakened version of the accommodation inequality (1). Here, $D \Psi(G)$ denotes the derivative of the response function $\Psi$. Because of the definition (3) of the disarrangement tensor $M$, the system (5) - (7) amounts to thirteen scalar relations to determine the twelve scalar fields that characterize $g$ and $G$. The stress tensor $S$ in the reference configuration is determined in the present case of purely dissipative disarrangements through the stress relation

$$
S=D \Psi
$$

and this relation then permits one to impose boundary conditions of place and/or of traction in connection with the system (5) - (7). (As in the context of classical, non-linear elasticity, the assumption that the free energy response function $\Psi$ is frame indifferent implies that balance of angular momentum is 
satisfied.)

The significance of the consistency relation (6) in the present study is underscored by the following result $[20,21]$ which shows that, under mild assumptions on the free energy response function $\Psi$, rank-one disarrangements associated with a structured deformation $(g, G)$ that satisfies the consistency relation (6) generally decrease the free energy from its value for the corresponding (classical) structured deformation $(g, \nabla g)$.

Remark 1 Assume that the free energy response function $\Psi$ not only is smooth but also is rank-one convex, i.e., for all tensors $A$ and vectors $a$ and $b$ such that both $\operatorname{det} A$ and $\operatorname{det}(A+a \otimes b)$ are positive, there holds

$$
D \Psi(A) \cdot(a \otimes b) \leq \Psi(A+a \otimes b)-\Psi(A) .
$$

Let $(g, G)$ a structured deformation and $X$ a point in the body be given such that the disarrangement tensor $M(X)=\nabla g(X)-G(X)$ has rank one and such that the consistency relation (6) is satisfied. It follows that the free energy density $\Psi(G(X))$ at $X$ for the structured deformation $(g, G)$ is no greater than the free energy density $\Psi(\nabla g(X))$ at $X$ for the classical deformation $(g, \nabla g)$ :

$$
\Psi(G(X)) \leq \Psi(\nabla g(X)) .
$$

\subsection{Disarrangement phases}

Among the field relations (5) - (7) above, we focus attention on the consistency relation (6) that, at each point $X$ in the body, requires that the deformation without disarrangements $G(X)$ and the macroscopic deformation gradient $F(X):=\nabla g(X)$ satisfy

$$
D \Psi(G(X))\left(F(X)^{T}-G(X)^{T}\right)=0,
$$


and on the accommodation inequality (7)

$$
0<\operatorname{det} G(X) \leq \operatorname{det} F(X)
$$

If we consider a given material point $X$ and omit from our notation the dependence upon $X$, then these relations amount to the following pair of requirements to be satisfied by tensors $F$ and $G$ :

$$
D \Psi(G)\left(F^{T}-G^{T}\right)=0 \text { and } 0<\operatorname{det} G \leq \operatorname{det} F .
$$

For a given tensor $F$, we call a tensor $G$ that satisfies both relations in (13) a disarrangement phase corresponding to $F$ for the aggregate $[20,21]$. Once the tensor $F$ is given, each disarrangement phase $G$ corresponding to $F$ may be thought of as a state of deformation in which the aggregate itself undergoes the homogeneous deformation $X \longmapsto X_{0}+F\left(X-X_{0}\right)$ and in which each piece undergoes the homogeneous deformation $X \longmapsto X_{0}+G\left(X-X_{0}\right)$.

For every choice of free energy response function $\Psi$ and for every choice of macroscopic deformation gradient $F$, the choice $G=F$ satisfies both the relations in (13), and we call the resulting disarrangement phase $G=F$ the compact phase corresponding to $F$ [7]. In the compact phase, $M$ is zero, so that there are no disarrangements, and each piece of the aggregate deforms in the same way as the aggregate itself.

For a second example of disarrangement phases, we showed [7] that, for a broad class of isotropic free energy response functions $\Psi$ satisfying standard semiconvexity and growth properties, there exists a positive number $\varsigma_{\min }$ such that $\Psi$ attains an absolute minimum at each tensor $\varsigma_{\min } R$ with $R$ a rotation tensor. Consequently, $D \Psi\left(\varsigma_{\min } R\right)=0$ so that for every choice of $F$ the consistency relation $(13)_{1}$ is satisfied with $G=\varsigma_{\min } R$. In order that the the accommodation 
inequality $(13)_{2}$ also be satisfied for this choice of $G$, we must have

$$
\varsigma_{\min }^{3} \leq \operatorname{det} F
$$

Therefore, if $F$ satisfies (14), then for each rotation tensor $R$, the tensor $G=$ $\varsigma_{\min } R$ is a disarrangement phase corresponding to $F$. Because $D \Psi\left(\varsigma_{\min } R\right)=0$ each piece of the aggregate is stress-free in such a phase. Consequently, this disarrangement phase describes the aggregate in a state in which the macroscopic deformation provides via the inequality (14) enough room for each piece of the aggregate to deform into a stress-free configuration in which all the principal stretches are equal to $\varsigma_{\min }$ and to rotate via $R$. Thus, each piece of the aggregate in this phase is completely relaxed, and we call $\varsigma_{\min } R$ the loose phase corresponding to $F$ and $R$.

\subsection{Stable disarrangement phases}

The examples available in the literature $[2,3,7,20,21]$ show that, given the free-energy response function $\Psi$ and the macroscopic deformation gradient $F$, there are many disarrangement phases corresponding to $F$. The multiplicity of disarrangement phases $G$ corresponding to a given $F$ appearing in the different contexts suggested additional conditions for selecting preferred disarrangement phases.

In the present context of statics, an appropriate notion of material stability was introduced in $[20,21]$ : for a given macroscopic deformation gradient $F$, a tensor $G$ is called a stable disarrangement phase corresponding to $F$ if, not only is $G$ a disarrangement phase corresponding to $F$, but also $G$ delivers the minimum energy density $\Psi\left(G^{\prime}\right)$ among all disarrangement phases $G^{\prime}$ corresponding to $F$. Thus, each stable disarrangement phase $G$ corresponding to $F$ is a solution to 
the minimization problem :

$$
\min _{G^{\prime}} \Psi\left(G^{\prime}\right) \quad \text { subject to } \quad 0<\operatorname{det} G^{\prime} \leq \operatorname{det} F \text { and } \quad D \Psi\left(G^{\prime}\right)\left(F^{T}-G^{\prime T}\right)=0 \text {. }
$$

For example, in the context for the notion of "loose phase" described in the previous section, for each rotation $R$ and each tensor $F$ satisfying (14), the tensor $\varsigma_{\min } R$ is a stable disarrangement phase corresponding to $F$, because $G^{\prime}=\varsigma_{\min } R$ is an absolute minimizer of the free energy response function and satisfies both relations in (15). Of course, if the tensor $F$ does not satisfy the inequality (14), then there is no loose phase corresponding to $F$ and $R$, no matter what the choice of rotation tensor $R$. Turning to the notion of "compact phase," we note that, while the tensor $G=F$ always is available as the compact phase corresponding to $F$, this compact phase need not be a stable disarrangement phase corresponding to $F$, since $G=F$ need not minimize the energy among disarrangement phases $G^{\prime}$ corresponding to $F$ and, therefore, need not be a solution of the problem (15). Thus, for arbitrary macroscopic deformation gradients $F$, the compact phase corresponding to $F$ always competes for the status of a stable disarrangement phase but need not win that status. By contrast, only for $F$ satisfying $\varsigma_{\min }^{3} \leq \operatorname{det} F$ is the loose phase $\varsigma_{\min } R$ a competitor; however, when it does compete, the loose phase always achieves the status of stable disarrangement phase.

Remark 1 and the notion of stable disarrangement phases corresponding to $F$ now tell us: if the compact phase for $F$ is a stable disarrangement phase corresponding to $F$, then so are all disarrangement phases $G$ for $F$ having $F-G$ of rank one. 


\section{$2.3 \quad$ A model free energy $\Psi_{\alpha \beta}$}

In order to illustrate the richness of possibilities for disarrangement phases of aggregates of elastic bodies, we chose in [7] and in [20, 21] a specific free energy response function that appears widely in the literature. We let $\alpha$ and $\beta$ be positive numbers and consider henceforth a granular medium whose free energy response function is

$$
\Psi_{\alpha \beta}(G)=\frac{1}{2} \alpha(\operatorname{det} G)^{-2}+\frac{1}{2} \beta \operatorname{tr}\left(G G^{T}\right)=\frac{1}{2} \beta\left(\frac{r}{\operatorname{det} B_{G}}+\operatorname{tr} B_{G}\right)
$$

where $B_{G}:=G G^{T}$ is a Cauchy-Green tensor corresponding to $G$ and $r:=\alpha / \beta$. Here, the numbers $\alpha$ and $\beta$ represent "elastic constants" for the pieces of the aggregate, and they determine the stress response in the reference configuration through the relation

$$
\beta^{-1} S=\beta^{-1} D \Psi_{\alpha \beta}(G)=-\frac{r}{(\operatorname{det} G)^{2}} G^{-T}+G .
$$

It is easy to verify from the previous two relations that not only is the free energy $\Psi_{\alpha \beta}$ rank-one convex (9), but also is strictly rank-one convex, in the sense that equality holds in (9) if and only if $a=0$ or $b=0$. Rank-one convexity of $\Psi_{\alpha \beta}$ along with its smoothness imply [19] that $\Psi_{\alpha \beta}$ satisfies the Legendre-Hadamard condition: for all $G$ with $\operatorname{det} G>0$ and for all $c, d \in \mathcal{V}$

$$
D^{2} \Psi_{\alpha \beta}(G) c \otimes d \cdot c \otimes d \geq 0 .
$$

Consequently, the lack of stability for any of the particular disarrangement phases considered in the sequel cannot be attributed to failure of the LegendreHadamard condition (18). 
We note for this model aggregate that $D \Psi_{\alpha \beta}(G)=0$ if and only if

$$
\frac{r}{(\operatorname{det} G)^{2}} G^{-T}=G
$$

Writing $G=V_{G} R_{G}$ in its polar decomposition (with $V_{G}$ symmetric and positive definite and $R_{G}$ a rotation) this relation becomes $V_{G}^{2}=r\left(\operatorname{det} V_{G}\right)^{-2} I$, with $I$ the identity tensor, so that $V_{G}=\sqrt{r}\left(\operatorname{det} V_{G}\right)^{-1} I$. Taking the determinant of both sides tells us that $\operatorname{det} V_{G}=r^{3 / 8}$. Therefore, $V_{G}=r^{1 / 8} I$, and we may conclude:

$D \Psi_{\alpha \beta}(G)=0 \quad$ if and only if $G=r^{1 / 8} R$ for some rotation $R$.

Thus, the only candidates for stationary points for the free energy response are $G=r^{1 / 8} R$ with $R$ a rotation, and the free energy (16) at such points is given by

$$
\frac{2}{\beta} \Psi_{\alpha \beta}\left(r^{1 / 8} R\right)=\frac{r}{r^{3 / 4}}+\operatorname{tr}\left(r^{1 / 4} I\right)=4 r^{1 / 4}
$$

The growth properties of $\Psi_{\alpha \beta}$ as $\operatorname{det} G$ tends to zero and as $\operatorname{tr}\left(G G^{T}\right)$ tends to infinity tell us that $\frac{2}{\beta} \Psi_{\alpha \beta}$ attains the absolute minimum value $4 r^{1 / 4}$ at precisely the points $G=r^{1 / 8} R$ with $R$ a rotation. From the discussion preceding (14) we conclude that for this free energy, $\zeta_{\min }=r^{1 / 8}$. Consequently, for each macroscopic deformation gradient $F$ satisfying

$$
r^{3 / 8}=\operatorname{det}\left(r^{1 / 8} R\right) \leq \operatorname{det} F
$$

the tensors $G=r^{1 / 8} R$ are the loose phases corresponding to $F$. In fact, for every macroscopic deformation field $g$ that satisfies $r^{3 / 8} \leq \operatorname{det} \nabla g(X)$ for all $X$ in the body, and for every choice of rotation field $X \longmapsto Q(X)$ on the body, the structured deformation $\left(g, r^{1 / 8} Q\right)$ has the property that, at every point $X$ 
in the body, $G(X)$ is a loose phase corresponding to $\nabla g(X)$. Moreover, this family of structured deformations includes all possibilities for achieving loose phases in the aggregate. The fact that the field $G$ need not itself be a gradient tells us that the rotation field $Q$ can vary from point to point. Therefore, the loose phases can support a texturing at the length scale of the individual pieces of the aggregate.

For each macroscopic deformation gradient $F$, the compact phase $G=F$ corresponding to $F$ yields the stress in the reference configuration $S$ satisfying

$$
\beta^{-1} S=F-\frac{r}{(\operatorname{det} F)^{2}} F^{-T}
$$

as well as the stress in the deformed configuration $T$ satisfying

$$
\begin{aligned}
\beta^{-1}(\operatorname{det} F) T & =\beta^{-1} S F^{T}=F F^{T}-r\left(\operatorname{det}\left(F F^{T}\right)\right)^{-1} I \\
& =B_{F}-r\left(\operatorname{det} B_{F}\right)^{-1} I
\end{aligned}
$$

with $B_{F}=F F^{T}$.

\subsection{General solutions of the consistency relation associ- ated with $\Psi_{\alpha \beta}$}

With a view toward determining the stable disarrangement phases of the model granular medium, we determined in $[20,21]$ all of the solutions of the consistency relation $(13)_{1}$, which here, by $(22)$, is equivalent to

$$
\left(G-\frac{r}{(\operatorname{det} G)^{2}} G^{-T}\right)\left(F^{T}-G^{T}\right)=0 .
$$

Specifically, we let $F$ be given and seek all solutions $G$ with $\operatorname{det} G>0$ of (24), without for the moment taking into account satisfaction of the accommodation 
inequality $(13)_{2}$. Using again the polar decomposition $G=V_{G} R_{G}$ and the Cauchy-Green tensor $B_{G}=G G^{T}=V_{G}^{2}$, we may write (24) in the equivalent form

$$
\left(V_{G}-\frac{r}{\left(\operatorname{det} V_{G}\right)^{2}} V_{G}^{-1}\right)\left(R_{G} F^{T}-V_{G}\right)=0
$$

or, by multiplying the last relation on the left by $V_{G}$, in the form

$$
\left(B_{G}-\frac{r}{\operatorname{det} B_{G}} I\right)\left(R_{G} F^{T}-V_{G}\right)=0 .
$$

\subsubsection{The case $G=F$ (compact phase)}

We first consider the case $G=F$ (considered above in the discussion of the compact phase corresponding to $F$ ), so that the expression $R_{G} F^{T}-V_{G}$ equals $R_{F} F^{T}-V_{F}=0$. Consequently, the consistency relation (25) is satisfied in this case, and we have the following expressions for the Cauchy stress $T=$ $(\operatorname{det} F)^{-1} D \Psi_{\alpha \beta}(G) F^{T}$ and for the free energy $\Psi_{\alpha \beta}(G)$ :

$$
\begin{aligned}
\beta^{-1}(\operatorname{det} F) T & =F F^{T}-\frac{r}{(\operatorname{det} F)^{2}} I \\
2 \beta^{-1} \Psi_{\alpha \beta}(G) & =\frac{r}{(\operatorname{det} F)^{2}}+\operatorname{tr}\left(F F^{T}\right)
\end{aligned}
$$

Of course, in this case the accommodation inequality $(13)_{2}$ is satisfied with equality.

\subsubsection{The case $G \neq F \quad$ (non-compact phases)}

We assume now that $G \neq F$ and note from (25) that the range of $R_{G} F^{T}-V_{G}$ then contains non-zero elements and, hence, the nullspace of $B_{G}-\frac{r}{\operatorname{det} B_{G}} I$ is nontrivial. Consequently, the number $r / \operatorname{det} B_{G}$ must be one of the eigenvalues $\lambda_{1}^{G}$, $\lambda_{2}^{G}, \lambda_{3}^{G}$ of $B_{G}$, say (without loss of generality) $\lambda_{1}^{G}$ and, since $\operatorname{det} B_{G}=\lambda_{1}^{G} \lambda_{2}^{G} \lambda_{3}^{G}$, 
we have

$$
\left(\lambda_{1}^{G}\right)^{2} \lambda_{2}^{G} \lambda_{3}^{G}=r
$$

At this point we invoke the Spectral Theorem to represent $V_{G}$ and $B_{G}=V_{G}^{2}$ in terms of an orthonormal basis $e_{1}^{G}, e_{2}^{G}, e_{3}^{G}$ of eigenvectors corresponding to the eigenvalues $\lambda_{1}^{G}, \lambda_{2}^{G}, \lambda_{3}^{G}$ of $B_{G}$ :

$$
B_{G}=\sum_{i=1}^{3} \lambda_{i}^{G} e_{i}^{G} \otimes e_{i}^{G} \text { and } V_{G}=\sum_{i=1}^{3}\left(\lambda_{i}^{G}\right)^{1 / 2} e_{i}^{G} \otimes e_{i}^{G} .
$$

We assume without loss of generality that $e_{1}^{G}=e_{2}^{G} \times e_{3}^{G}$, and, substituting these expressions for $B_{G}$ and $V_{G}$ into (25), taking into account (28), and using $I=\sum_{i=1}^{3} e_{i}^{G} \otimes e_{i}^{G}$ we find that the consistency relation is equivalent to to the system of vector relations

$$
\left(\lambda_{i}^{G}-\lambda_{1}^{G}\right)\left(F R_{G}^{T}-\left(\lambda_{i}^{G}\right)^{1 / 2} I\right) e_{i}^{G}=0 \quad \text { for } \quad i=2,3,
$$

The case $\left(\lambda_{1}^{G}\right)^{2} \lambda_{2}^{G} \lambda_{3}^{G}=r$ and $\lambda_{i}^{G} \neq \lambda_{1}^{G}$ for $i=2,3$ ("plane-stress") In view of (30) we conclude in this case that the consistency relation is equivalent to the relations

$$
R_{G}^{T} e_{i}^{G}=\left(\lambda_{i}^{G}\right)^{1 / 2} F^{-1} e_{i}^{G} \quad \text { for } \quad i=2,3
$$

In $[20,21]$ we established the following characterization of solutions of the consistency relation (31) in the present case:

Remark 2 Let orthogonal unit vectors $e$ and $f$ and a linear mapping $F$ with 
$\operatorname{det} F>0$ be given satisfying

$$
F^{-1} e \cdot F^{-1} f=0, \quad r^{1 / 2}\left|F^{-1} e\right|^{3}\left|F^{-1} f\right| \neq 1, \quad r^{1 / 2}\left|F^{-1} e\right|\left|F^{-1} f\right|^{3} \neq 1 .
$$

Then the tensor

$$
\begin{aligned}
G= & r^{1 / 4}\left|F^{-1} e\right|^{1 / 2}\left|F^{-1} f\right|^{1 / 2}(e \times f) \otimes\left(\frac{F^{-1} e}{\left|F^{-1} e\right|} \times \frac{F^{-1} f}{\left|F^{-1} f\right|}\right)+ \\
& +\left|F^{-1} e\right|^{-1} e \otimes \frac{F^{-1} e}{\left|F^{-1} e\right|}+\left|F^{-1} f\right|^{-1} f \otimes \frac{F^{-1} f}{\left|F^{-1} f\right|}
\end{aligned}
$$

is a solution of the consistency relation (31), and the solution (33) equals $F$ if and only if

$$
B_{F}(e \times f)=\frac{r}{\operatorname{det} B_{F}} e \times f
$$

Moreover, every solution $G \neq F$ of the consistency relation (31) in the case $\lambda_{i}^{G} \neq \lambda_{1}^{G}$ for $i=2,3$ is of the form (33) for some choice of the orthogonal unit vectors $e$ and $f$ satisfying (32), and this formula for $G$ implies that

$$
\begin{gathered}
V_{G}=r^{1 / 4}\left|F^{-1} e\right|^{1 / 2}\left|F^{-1} f\right|^{1 / 2}(e \times f) \otimes(e \times f)+ \\
+\left|F^{-1} e\right|^{-1} e \otimes e+\left|F^{-1} f\right|^{-1} f \otimes f \\
R_{G}=(e \times f) \otimes\left(\frac{F^{-1} e}{\left|F^{-1} e\right|} \times \frac{F^{-1} f}{\left|F^{-1} f\right|}\right)+ \\
\quad+e \otimes \frac{F^{-1} e}{\left|F^{-1} e\right|}+f \otimes \frac{F^{-1} f}{\left|F^{-1} f\right|} \\
\operatorname{det} G=r^{1 / 4}\left|F^{-1} e\right|^{-1 / 2}\left|F^{-1} f\right|^{-1 / 2}
\end{gathered}
$$

In addition, if $T=(\operatorname{det} F)^{-1} D \Psi_{\alpha \beta}(G) F^{T}$ is the Cauchy stress, then

$$
\beta^{-1}(\operatorname{det} F) T=\left|F^{-1} e\right|^{-2}\left(1-r^{1 / 2}\left|F^{-1} e\right|^{3}\left|F^{-1} f\right|\right) e \otimes e+
$$




$$
+\left|F^{-1} f\right|^{-2}\left(1-r^{1 / 2}\left|F^{-1} e\right|\left|F^{-1} f\right|^{3}\right) f \otimes f,
$$

and the free energy $\Psi_{\alpha \beta}(G)$ is given by

$$
\frac{2}{\beta} \Psi_{\alpha \beta}(G)=2 r^{1 / 2}\left|F^{-1} e\right|\left|F^{-1} f\right|+\left|F^{-1} e\right|^{-2}+\left|F^{-1} f\right|^{-2} .
$$

The formula (38) for the Cauchy stress implies that the traction $T(e \times f)$ on a plane with normal $e \times f$ is zero and that every traction vector $T n$ lies in the plane determined by $e$ and $f$. Moreover, both $T e$ and $T f$ are non-zero. It is then appropriate to use the attribute plane-stress to describe the solutions $G$ in (33) of the consistency relation in the present case $\lambda_{i}^{G} \neq \lambda_{1}^{G}$ for $i=2,3$, and we use the term plane-stress disarrangement phases corresponding to $F$ in referring to such tensors $G$ that also satisfy the accommodation inequality (7) in the form $0<\operatorname{det} G \leq \operatorname{det} F$ :

$$
0<r^{1 / 4}\left|F^{-1} e\right|^{-1 / 2}\left|F^{-1} f\right|^{-1 / 2} \leq \operatorname{det} F .
$$

The case $\left(\lambda_{1}^{G}\right)^{2} \lambda_{2}^{G} \lambda_{3}^{G}=r$ and $\lambda_{2}^{G}=\lambda_{1}^{G}, \lambda_{3}^{G} \neq \lambda_{1}^{G}$ ("uniaxial stress") From (30) we have in this case that the consistency relation is equivalent to the single condition

$$
R_{G}^{T} e_{3}^{G}=\left(\lambda_{3}^{G}\right)^{1 / 2} F^{-1} e_{3}^{G},
$$

and the solutions of the consistency relation in this form were characterized in $[20,21]$ as follows:

Remark 3 Let a unit vector e, a proper orthogonal tensor $R$, and a linear 
mapping $F$ with $\operatorname{det} F>0$ be given satisfying

$$
R^{T} e=\frac{F^{-1} e}{\left|F^{-1} e\right|} \quad \text { and } \quad r^{1 / 8}\left|F^{-1} e\right| \neq 1
$$

Then the tensor $G$ given by

$$
G=r^{1 / 6}\left|F^{-1} e\right|^{1 / 3}(I-e \otimes e) R+\left|F^{-1} e\right|^{-1} e \otimes \frac{F^{-1} e}{\left|F^{-1} e\right|}
$$

is a solution of the consistency relation (30) for the case $\left(\lambda_{1}^{G}\right)^{2} \lambda_{2}^{G} \lambda_{3}^{G}=r$ and $\lambda_{2}^{G}=\lambda_{1}^{G}, \lambda_{3}^{G} \neq \lambda_{1}^{G}$. The solution $G$ in (43) equals $F$ if and only if $R_{F}=R$ and, for all vectors $v$ perpendicular to $e$,

$$
B_{F} v=\frac{r}{\operatorname{det} B_{F}} v
$$

Moreover, every solution of the consistency relation for this case is of the form (43) with $R$ and e satisfying (42), and the following relations hold:

$$
\begin{aligned}
V_{G} & =r^{1 / 6}\left|F^{-1} e\right|^{1 / 3}(I-e \otimes e)+\left|F^{-1} e\right|^{-1} e \otimes e \\
R_{G} & =R \\
\operatorname{det} G & =\operatorname{det} V_{G}=r^{1 / 3}\left|F^{-1} e\right|^{-1 / 3}
\end{aligned}
$$

In addition, if $T=(\operatorname{det} F)^{-1} D \Psi_{\alpha \beta}(G) F^{T}$ is the Cauchy stress, then

$$
\beta^{-1}(\operatorname{det} F) T=\frac{1-r^{1 / 3}\left|F^{-1} e\right|^{8 / 3}}{\left|F^{-1} e\right|^{2}} e \otimes e,
$$

and the free energy $\Psi_{\alpha \beta}(G)$ is given by

$$
\frac{2}{\beta} \Psi_{\alpha \beta}(G)=3 r^{1 / 3}\left|F^{-1} e\right|^{2 / 3}+\left|F^{-1} e\right|^{-2}
$$


The formula (48) and the restriction (42) show that the state of stress in the deformed configuration of the aggregate is uniaxial and non-zero for every solution $G$ of the consistency relation in the present case $\left(\lambda_{1}^{G}\right)^{2} \lambda_{2}^{G} \lambda_{3}^{G}=r$ and $\lambda_{2}^{G}=\lambda_{1}^{G}, \lambda_{3}^{G} \neq \lambda_{1}^{G}$. It is then appropriate to use the attribute uniaxial stress to describe the solutions $G$ and the term uniaxial stress disarrangement phases corresponding to $F$ in referring to such tensors $G$ that also satisfy the accommodation inequality $(15)_{2}$ in the form:

$$
0<r^{1 / 3}\left|F^{-1} e\right|^{-1 / 3} \leq \operatorname{det} F
$$

The case $\left(\lambda_{1}^{G}\right)^{2} \lambda_{2}^{G} \lambda_{3}^{G}=r$ and $\lambda_{1}^{G}=\lambda_{2}^{G}=\lambda_{3}^{G}$ ("zero stress"/loose phase) The relation (28) immediately yields $\lambda_{1}^{G}=\lambda_{2}^{G}=\lambda_{3}^{G}=r^{1 / 4}$, so that

$$
B_{G}=r^{1 / 4} I \text { and } G=r^{1 / 8} R,
$$

with no restriction on the rotation $R=R_{G}$ imposed by the consistency relation. Of course, in this case we also have

$$
\operatorname{det} G=r^{3 / 8}
$$

and we note that this case recovers precisely those tensors $G$ identified the previous section that render $\Psi_{\alpha \beta}$ a minimum and that enter into the description of the loose phase. We have from those considerations

$$
T=0 \quad \text { and } \quad 2 \beta^{-1} \Psi_{\alpha \beta}(G)=4 r^{1 / 4},
$$


and the accommodation inequality $(15)_{2}$ takes the form

$$
r^{3 / 8} \leq \operatorname{det} F
$$

\section{Stable disarrangement phases associated with $\Psi_{\alpha \beta}$ corresponding to $F=\lambda^{1 / 2} I$}

For each positive number $\lambda$ we consider the tensor $F=\lambda^{1 / 2} I$ representing, for $\lambda>1$, the gradient of a uniform expansion about a given point and, for $\lambda<1$, the gradient of a uniform contraction. The disarrangement phases listed below are obtained by substitution of $F=\lambda^{1 / 2} I$ into each of the categories of disarrangement phases for general $F$ obtained earlier.

Compact disarrangement phase when $F=\lambda^{1 / 2} I$

$$
\begin{aligned}
G & =\lambda^{1 / 2} I \\
\lambda^{3 / 2} & =\lambda^{3 / 2} \quad(\text { accommodation inequality }) \\
\frac{2}{\beta} \Psi_{\alpha \beta}(G) & =r \lambda^{-3}+3 \lambda \\
\frac{1}{\beta} T & =\lambda^{-1 / 2}\left(1-r \lambda^{-4}\right) I \\
M & =0
\end{aligned}
$$

Plane-stress disarrangement phases when $F=\lambda^{1 / 2} I$

$$
\begin{gathered}
G=r^{1 / 4} \lambda^{-1 / 2}(e \times f) \otimes(e \times f)+\lambda^{1 / 2}(I-(e \times f) \otimes(e \times f)) \\
r^{1 / 4} \leq \lambda \quad(\text { accommodation inequality }) \\
\frac{2}{\beta} \Psi_{\alpha \beta}(G)=2 r^{1 / 2} \lambda^{-1}+2 \lambda,
\end{gathered}
$$




$$
\begin{gathered}
\frac{1}{\beta} T=\lambda^{-1 / 2}\left(1-r^{1 / 2} \lambda^{-2}\right)(I-(e \times f) \otimes(e \times f)), \\
M=\lambda^{1 / 2}\left(1-r^{1 / 4} \lambda^{-1}\right)(e \times f) \otimes(e \times f) \\
r^{1 / 4} \neq \lambda .
\end{gathered}
$$

Uniaxial stress disarrangement phases when $F=\lambda^{1 / 2} I$

$$
\begin{gathered}
G=\left\{r^{1 / 6} \lambda^{-1 / 6} I+\lambda^{1 / 2}\left(1-r^{1 / 6} \lambda^{-2 / 3}\right) e \otimes e\right\} R \\
r^{1 / 4} \leq \lambda \quad(\text { accommodation inequality }) \\
\frac{2}{\beta} \Psi_{\alpha \beta}(G)=3 r^{1 / 3} \lambda^{-1 / 3}+\lambda, \\
\frac{1}{\beta} T=\lambda^{-1 / 2}\left(1-r^{1 / 3} \lambda^{-4 / 3}\right) e \otimes e \\
M=\left(\lambda^{1 / 2} I-r^{1 / 6} \lambda^{-1 / 6} R\right)(I-e \otimes e) \\
R e=e, \quad r^{1 / 4} \neq \lambda .
\end{gathered}
$$

Loose disarrangement phases when $F=\lambda^{1 / 2} I$

$$
\begin{gathered}
G=r^{1 / 8} R \\
r^{1 / 4} \leq \lambda \quad \text { (accommodation inequality) } \\
\frac{2}{\beta} \Psi_{\alpha \beta}(G)=4 r^{1 / 4} \\
T=0 \\
M=\lambda^{1 / 2} I-r^{1 / 8} R
\end{gathered}
$$


For all but the compact phase, the accommodation inequality takes the form $r^{1 / 4} \leq \lambda$ and implies that the compact phase is the only competitor for stability when $\lambda<r^{1 / 4}$. Consequently, the compact phase $G=\lambda^{1 / 2} I$ is the only stable disarrangement phase corresponding to $F=\lambda^{1 / 2} I \quad$ when the extension parameter $\lambda$ lies in the interval $\left(0, r^{1 / 4}\right)$. For $r^{1 / 4}=\lambda$ only the loose phase $G=r^{1 / 8} R_{G}$ and the compact phase $G=F$ compete, and they yield the same value $4 r^{1 / 4}$ of $\frac{2}{\beta} \Psi_{\alpha \beta}$. Therefore, both the loose phase $G=r^{1 / 8} R_{G}$ and the compact phase $G=F$ are stable disarrangement phases for $F=\lambda^{1 / 2} I$ when $r^{1 / 4}=\lambda$. For $\lambda$ in the interval $\left(r^{1 / 4}, \infty\right)$ all four categories of phases compete for stability. The relation (19) shows that, in the loose phase $G=r^{1 / 8} R_{G}$, and in the loose phase alone, $\frac{2}{\beta} \Psi_{\alpha \beta}$ attains its minimum value $4 r^{1 / 4}$, so that only the loose phase $G=r^{1 / 8} R$ is a stable disarrangement phase corresponding to $F=\lambda^{1 / 2} I$ for $\lambda$ in the interval $\left(r^{1 / 4}, \infty\right)$. Figure 2 shows in the $\lambda-r$ plane the stable disarrangement phases that are available corresponding to the uniform expansion/contraction $F=\lambda^{1 / 2} I$. While we have considered until now the ratio $r=\alpha / \beta$ as fixed, we can view the different values of $r$ depicted in the figure as accessible via changes in the temperature (provided by the dependence of the elastic constants $\alpha$ and $\beta$ on temperature), or accessible via replacement of a given material with response $\Psi_{\alpha \beta}$ by another with response $\Psi_{\alpha^{\prime}, \beta^{\prime}}$ with $\alpha^{\prime} / \beta^{\prime} \neq \alpha / \beta$.

We note that on the curve $\lambda=r^{1 / 4}$ the two stable disarrangement phases (the compact and the loose phases) not only have the same free energy density $2 \beta r^{1 / 4}=2 \alpha^{1 / 4} \beta^{3 / 4}$ but also both have the same stress $T=0$. However, when $\lambda=r^{1 / 4}$ and $R \neq I$, the disarrangement tensors $M=0$ in the compact phase and $M=\lambda^{1 / 2} I-r^{1 / 8} R=r^{1 / 8}(I-R)$ in the loose phase are different, so that one cannot simply coalesce the two stable phases into one on the curve $\lambda=r^{1 / 4}$. 


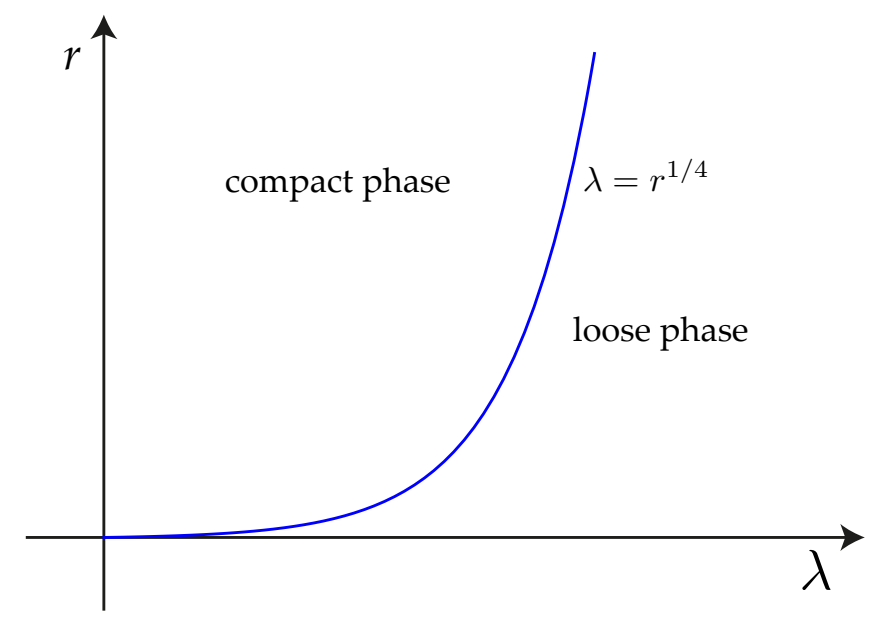

Figure 2: Stable disarrangement phases for $F=\lambda^{1 / 2} I$

For a given $r>0$, when the extension parameter $\lambda$ lies in the interval $\left(0, r^{1 / 4}\right)$ in which the compact phase is stable, then the stress $T=\beta \lambda^{-1 / 2}\left(1-r \lambda^{-4}\right) I$ is compressive, i.e., $T n \cdot n<0$ for every vector $n$. Alternatively, when the extension parameter $\lambda$ lies in the interval $\left(r^{1 / 4}, \infty\right)$ in which the loose phase is stable, then stress $T$ vanishes. Consequently, for the stable disarrangement phases corresponding to $F=\lambda^{1 / 2} I$, the stress either is compressive or vanishes. Consequently, the stress response under uniform expansions and contractions within the stable disarrangement phases is a non-linear analogue of a "no-tension" material response [4], [5], [6].

At the present stage of our research we view the profile of stable disarrangement phases provided in Figure 2 as a statical landscape of preferred structured deformations available to the material as it expands or contracts. While it is tempting to do so, we do not at this time view the profile of stable disarrangement phases as a guide to the time evolution of changes of state as the expansion parameter $\lambda$ varies with the ratio $r$ fixed (or, alternatively, as the temperature varies with $\lambda$ fixed). Further studies of the evolution of aggregates 
of elastic bodies $\backslash$ in the present framework are required to determine whether or not the profile of stable disarrangement phases also provides a guide to behavior in environments that vary with time.

\section{Stable disarrangement phases associated with $\Psi_{\alpha \beta}$ corresponding to $F=I+\mu a \otimes b$}

In this section we consider the family of simple shears generated by fixing a pair $a$ and $b$ of orthogonal unit vectors that determine the plane of shearing and, for each real number $\mu$, by taking

$$
F=I+\mu a \otimes b
$$

We then have

$$
\begin{aligned}
B_{F} & =F F^{T}=(I+\mu a \otimes b)(I+\mu a \otimes b)^{T} \\
& =I+\mu(a \otimes b+b \otimes a)+\mu^{2} a \otimes a,
\end{aligned}
$$

whose eigenvalues are the number 1 together with largest principal stretch $\lambda$ and its reciprocal:

$$
\begin{aligned}
\lambda & :=1+\frac{\mu^{2}+|\mu|\left(\mu^{2}+4\right)^{1 / 2}}{2} \geq 1, \\
\lambda^{-1} & =1+\frac{\mu^{2}-|\mu|\left(\mu^{2}+4\right)^{1 / 2}}{2} \leq 1,
\end{aligned}
$$

Since the amount of shear $\mu$ can be recovered from the formula

$$
\mu= \pm\left(\lambda+\lambda^{-1}-2\right)^{1 / 2}= \pm \frac{|\lambda-1|}{\lambda^{1 / 2}}
$$


it is convenient to use the largest principal stretch $\lambda \geq 1$ to parameterize the family of simple shears. In particular, for $\lambda>1$ the principal directions of stretch $e_{1}^{F}, e_{2}^{F}$, and $e_{3}^{F}$ corresponding, respectively, to $\lambda>1>\lambda^{-1}$ are given by

$$
\begin{aligned}
e_{1}^{F} & =\frac{\lambda^{1 / 2}}{(\lambda+1)^{1 / 2}} a+\frac{1}{(\lambda+1)^{1 / 2}} b, \\
e_{2}^{F} & =a \times b \\
e_{3}^{F} & =-\frac{1}{(\lambda+1)^{1 / 2}} a+\frac{\lambda^{1 / 2}}{(\lambda+1)^{1 / 2}} b,
\end{aligned}
$$

and we restrict our attention henceforth to the non-trival case $\lambda>1$. Consequently, we can write

$$
\begin{gathered}
B_{F}=\lambda e_{1}^{F} \otimes e_{1}^{F}+e_{2}^{F} \otimes e_{2}^{F}+\lambda^{-1} e_{3}^{F} \otimes e_{3}^{F} \\
\operatorname{det} F=\operatorname{det} B_{F}=1, \quad \operatorname{tr} B_{F}=\lambda+\lambda^{-1}+1 \\
F=V_{F} R_{F}=\left(\lambda^{1 / 2} e_{1}^{F} \otimes e_{1}^{F}+e_{2}^{F} \otimes e_{2}^{F}+\lambda^{-1 / 2} e_{3}^{F} \otimes e_{3}^{F}\right) R_{F},
\end{gathered}
$$

keeping in mind that $e_{1}^{F}, e_{2}^{F}$, and $e_{3}^{F}$ depend upon $\lambda$ according to (58). A simple computation yields expressions for $V_{F}$ and $R_{F}$ in terms of the original shearing vectors $a$ and $b$ and the principal stretch $\lambda$ :

$$
\begin{aligned}
& V_{F}=\frac{\lambda^{2}+1}{\lambda^{1 / 2}(\lambda+1)} a \otimes a+\frac{2 \lambda^{1 / 2}}{\lambda+1} b \otimes b+\frac{\lambda-1}{\lambda+1}(a \otimes b+b \otimes a)+ \\
& +(a \times b) \otimes(a \times b) \\
& R_{F}=\frac{2 \lambda^{1 / 2}}{\lambda+1}(a \otimes a+b \otimes b)+\frac{\lambda-1}{\lambda+1}(a \otimes b-b \otimes a)+ \\
& +(a \times b) \otimes(a \times b) .
\end{aligned}
$$


In particular, the tensor $R_{F}$ is a rotation about $a \times b$ by an angle $\theta_{F}$ determined by

$$
\cos \theta_{F}=\frac{2 \lambda^{1 / 2}}{\lambda+1}, \quad \sin \theta_{F}=\frac{\lambda-1}{\lambda+1}
$$

Moreover, we have for $i=1,2,3$ :

$$
\begin{aligned}
B_{F}^{-1} e_{i}^{G} \cdot e_{i}^{G} & =\left(\lambda^{-1} e_{1}^{F} \otimes e_{1}^{F}+e_{2}^{F} \otimes e_{2}^{F}+\lambda e_{3}^{F} \otimes e_{3}^{F}\right) e_{i}^{G} \cdot e_{i}^{G} \\
& =\lambda^{-1}\left(e_{1}^{F} \cdot e_{i}^{G}\right)^{2}+\left(e_{2}^{F} \cdot e_{i}^{G}\right)^{2}+\lambda\left(e_{3}^{F} \cdot e_{i}^{G}\right)^{2}
\end{aligned}
$$

and this quadratic form will determine the nature of the stable disarrangement phases corresponding to simple shear.

\subsection{Candidate for stability for the compact phase when}

$$
F=I+\mu a \otimes b
$$

For the compact phase corresponding to $F=I+\mu a \otimes b$, the results for general $F$ in Section 2 yield the following information:

$$
\begin{aligned}
G & =F=I+\mu a \otimes b \\
1 & =1 \quad \text { (accomodation inequality) } \\
\frac{2}{\beta} \Psi_{\alpha \beta}(G) & =r+1+\lambda+\lambda^{-1} \\
\frac{1}{\beta} T & =(\lambda-r) e_{1}^{F} \otimes e_{1}^{F}+(1-r) e_{2}^{F} \otimes e_{2}^{F}+\left(\lambda^{-1}-r\right) e_{3}^{F} \otimes e_{3}^{F} \\
M & =0
\end{aligned}
$$

In particular, there is only one compact disarrangement phase $G=I+\mu a \otimes b$ corresponding to $F=I+\mu a \otimes b$ and, hence, only one competitor for stability for the given value of shear $\mu$. Moreover, the compact phase arises no matter 
what the values of $\lambda>1$ and $r>0$, in contrast to the candidates for stability among plane-stress phases identified in the next subsection.

\subsection{Candidates for stability among plane-stress disarrange- ment phases when $F=I+\mu a \otimes b$}

The description of plane-stress disarrangement phases $G$ for general $F$ in Remark 2 includes the formula (39) for $\Psi_{\alpha \beta}(G)$ in the plane-stress category as well as restrictions on the vectors $e$ and $f$ in that formula that determine $G$ via (43). According to our discussion of stable disarrangement phases in Section 2, we can find candidates for stable plane-stress disarrangement phases by minimizing the function

$$
(e, f) \longmapsto H(e, f)=2 r^{1 / 2}\left|F^{-1} e\right|\left|F^{-1} f\right|+\left|F^{-1} e\right|^{-2}+\left|F^{-1} f\right|^{-2}
$$

subject to the constraints

$$
\begin{aligned}
e \cdot e & =f \cdot f=1, \quad e \cdot f=0, \quad F^{-1} e \cdot F^{-1} f=0 \\
r^{1 / 2} & \leq\left|F^{-1} e\right|\left|F^{-1} f\right| \\
1 & \neq r^{1 / 2}\left|F^{-1} e\right|^{3}\left|F^{-1} f\right| \\
1 & \neq r^{1 / 2}\left|F^{-1} e\right|\left|F^{-1} f\right|^{3} .
\end{aligned}
$$

Here, because $F=I+\mu a \otimes b$, the number $\operatorname{det} F=1$ no longer appears explicitly in the accommodation inequality (68).

The detailed steps in the solution of this minimization problem are provided in the appendix of [22], and they permit us to compare the energies among all of the stationary plane-stress disarrangement phases $G$ identified in that appendix. These comparisons are numerous, but elementary, and we include 
here only the conclusions obtained. Because different stationary phases arise in different regions of the $\lambda-r$ plane, the results of these comparisons are best viewed graphically in Figure 3. In three of the regions appearing in the figure, a formula for the minimum (normalized) energy $2 \beta^{-1} \Psi_{\alpha \beta}$ for stationary planestress phases is displayed, while in the region above the line $r=\lambda$ no minimum is recorded, because no stationary, plane-stress disarrangement phases arise for this region. (The appearance twice of the minimum value $2 r+1+r^{-1}$ is an indication that its set of competitors in the region $\lambda^{-1}<r<1$ is different from the set of competitors in the region $1 \leq r \leq \lambda$.) The lack of dependence upon $\lambda$ of the energy for these minima may be interpreted here as a decoupling of the energy stored in each piece of the aggregate from the amount of macroscopic shear experienced by the aggregate. It is interesting to note that, although the minimum values of energy in these plane-stress phases do not depend upon the stretch $\lambda$, other characteristics of these phases such as the stress may depend upon not only $\lambda$ but also on the principal directions of stretch for $G$, as we shall subsequently illustrate.

\subsection{Candidates for stability among uniaxial stress disar- rangement phases when $F=I+\mu a \otimes b$}

The description of plane-stress disarrangement phases $G$ for general $F$ in Remark 3 provides among other things the formula (49) for the free energy in the uniaxial stress category along with the restrictions (42) on the rotation tensor $R$ and the unit vector $e$ that appear in the representation formula (43) for $G$. Thus we are led here to minimize the function $J$ defined by

$$
e \longmapsto J(e)=3 r^{1 / 3}\left(B_{F}^{-1} e \cdot e\right)^{1 / 3}+\left(B_{F}^{-1} e \cdot e\right)^{-1}
$$






Figure 3: Minima of energy for plane-stress phases (simple shear).

subject to the constraints

$$
e \cdot e=1, \quad r^{2} \leq B_{F}^{-1} e \cdot e, \quad r^{1 / 4} B_{F}^{-1} e \cdot e \neq 1
$$

We have used the fact that $\operatorname{det} F=1$ in writing the accommodation inequality in the form $(72)_{2}$. Because the rotation tensor $R$ does not affect the value of the free energy, the restriction on $R$ contained in (42) need not be considered here. In the remainder of this subsection we provide candidates for minimizers of the function $J$ subject to the given constraints.

\subsubsection{Case $r^{2}=B_{F}^{-1} e \cdot e$}

In this case, the function $J$ can only take on the value $3 r^{1 / 3} r^{2 / 3}+r^{-2}=3 r+r^{-2}$, and the restriction $r^{1 / 4} B_{F}^{-1} e \cdot e \neq 1$ reduces to $r \neq 1$. The fact that $e$ is a unit 
vector in the present case implies the additional inequality constraints

$$
\lambda^{-1} \leq r^{2} \leq \lambda
$$

\subsubsection{Case $r^{2}<B_{F}^{-1} e \cdot e$}

We can find all the candidates $e$ for minimizers of $J$ by finding the stationary points of the function

$$
e \longmapsto \Xi(e)=3 r^{1 / 3}\left(B_{F}^{-1} e \cdot e\right)^{1 / 3}+\left(B_{F}^{-1} e \cdot e\right)^{-1}+\xi(e \cdot e-1)
$$

i.e., vectors $e$ that satisfy the constraints $(72)_{1,2,3}$, the second with strict inequality, as well as the stationarity condition

$$
0=D_{e} \Xi(e)=\left(r^{1 / 3}\left(B_{F}^{-1} e \cdot e\right)^{-2 / 3}-\left(B_{F}^{-1} e \cdot e\right)^{-2}\right) 2 B_{F}^{-1} e+2 \xi e .
$$

The constraint $(72)_{3}$ implies that the coefficient of $2 B_{F}^{-1} e$ does not vanish, and we conclude that the stationarity condition is equivalent to the requirement that $e$ be an eigenvector of $B_{F}^{-1}$. The three cases that then arise are listed in the table below:

\begin{tabular}{|c|c|c|c|c|}
\hline$e$ & $B_{F}^{-1} e \cdot e$ & $r^{2}<B_{F}^{-1} e \cdot e$ & $r^{1 / 4} B_{F}^{-1} e \cdot e \neq 1$ & $J(e)$ \\
\hline$e_{1}^{F}$ & $\lambda^{-1}$ & $r<\lambda^{-1 / 2}$ & $r \neq \lambda^{4}$ & $3 r^{1 / 3} \lambda^{-1 / 3}+\lambda$ \\
\hline$e_{2}^{F}$ & 1 & $r<1$ & $r \neq 1$ & $3 r^{1 / 3}+1$ \\
\hline$e_{3}^{F}$ & $\lambda$ & $r<\lambda^{1 / 2}$ & $r \neq \lambda^{-4}$ & $3 r^{1 / 3} \lambda^{1 / 3}+\lambda^{-1}$ \\
\hline
\end{tabular}

At this point, we are in a position to compare the energies among all of the stationary uniaxial stress disarrangement phases $G$ identified above. Again, we include here only the conclusions obtained through this comparison, and we view the results graphically in Figure 4. In three of the four regions depicted in the 
figure, a formula for the minimum (normalized) energy $2 \beta^{-1} \Psi_{\alpha \beta}$ for that region is given (within the category of uniaxial stress phases). No formula is given for the region above the curve $r=\lambda^{1 / 2}$, because there are no stationary phases in this category that arise in this region. (The appearance of the minimum value $1+3 r^{1 / 3}$ twice indicates that its set of competitors in the region $0<r<\lambda^{-1 / 2}$ is different from the set of competitors in the region $\lambda^{-1 / 2} \leq r<1$.) We note for future reference that the minimum value $3 r+r^{-2}$ in the region $1 \leq r \leq \lambda^{1 / 2}$ arises in the case $r^{2}=B_{F}^{-1} e \cdot e$, while the minimum value $1+3 r^{1 / 3}$ in the region $0<r<1$ arises in the case $r^{2}<B_{F}^{-1} e \cdot e, e=e_{2}^{F}$.

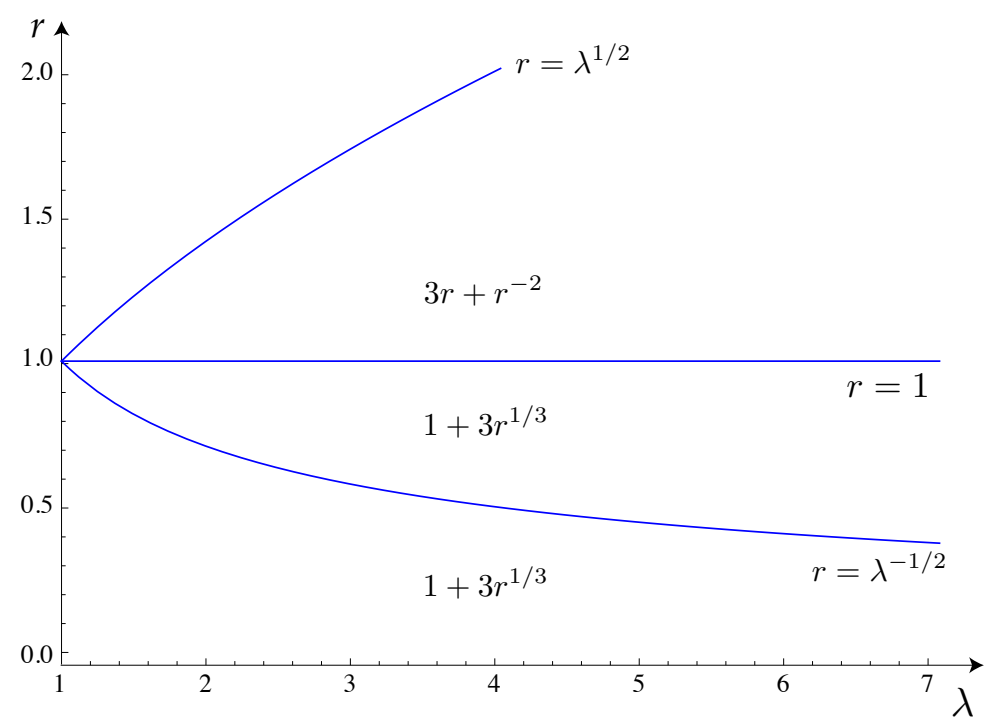

Figure 4: Minima of energy for uniaxial stress (simple shear). 


\subsection{Candidates for stability for the loose phases when $F=$ $I+\mu a \otimes b$}

Finally, the catalog of disarrangement phases under the category of loose phases yields the familiar formulas (with $\operatorname{det} F=1$ ):

$$
\begin{gathered}
G=r^{1 / 8} R_{G} \\
r \leq 1 \\
\frac{2}{\beta} \Psi_{\alpha \beta}(G)=4 r^{1 / 4},
\end{gathered}
$$

where $R_{G}$ is an arbitrary rotation. Every such disarrangement phase $G$ corresponds to the same (global minimizing) value of the energy.

\subsection{Stable disarrangement phases for simple shear}

The intracategory minima for $2 \beta^{-1} \Psi_{\alpha \beta}$ just obtained for the case of simple shear provide disarrangement phases for different categories that compete for the status of stable disarrangement phases in simple shear. From the candidates for stability obtained for the four categories in the previous subsections, we have the following regions in the quadrant $\lambda>1, r>0$ and intercategory competitors for stability:

- In the strip $\lambda>1,0<r<1$ the compact phase minimum $r+1+\lambda+\lambda^{-1}$, the plane-stress minimum $2 r^{1 / 2}+2$, the uniaxial stress minimum $3 r^{1 / 3}+1$, and the loose phase minimum $4 r^{1 / 4}$ all compete.

- In the region $\lambda>1,1<r<\lambda^{1 / 2}$ the compact phase minimum $r+1+$ $\lambda+\lambda^{-1}$, the plane-stress minium $2 r+1+r^{-1}$, and the uniaxial stress minimum $3 r+r^{-2}$ compete. 
- In the region $\lambda>1, \lambda^{1 / 2}<r<\lambda$ the compact phase minimum $r+1+$ $\lambda+\lambda^{-1}$ and the plane-stress minium $2 r+1+r^{-1}$ compete.

- In the region $\lambda>1, \lambda<r$ only the compact phase minimum $r+1+\lambda+\lambda^{-1}$ competes.

We undertake here the comparisons of the minima above in each region above, starting with the region listed last.

- For $\lambda>1, \lambda<r$, only the compact phase competes, so that the compact phase corresponding to $F=I+\mu a \otimes b$ is the stable disarrangement phase in this region.

- For $\lambda>1, \lambda^{1 / 2}<r<\lambda$, we note that the normalized energy minimum $2 \beta^{-1} \Psi_{\alpha \beta}^{c o m p}:=r+1+\lambda+\lambda^{-1}$ for the compact phase and the corresponding energy minimum $2 \beta^{-1} \Psi_{\alpha \beta}^{\text {plane }}:=2 r+1+r^{-1}$ for the plane-stress category of phases can be compared by comparing $\lambda+\lambda^{-1}$ and $r+r^{-1}$ on the given region. When $\lambda=r>1$, these two expressions are equal, and, because $\frac{d}{d \lambda}\left(\lambda+\lambda^{-1}\right)=1-\lambda^{-2}>0$, it follows that $\lambda+\lambda^{-1}>r+r^{-1}$ for $\lambda>r$, and, therefore, $\Psi_{\alpha \beta}^{c o m p}>\Psi_{\alpha \beta}^{\text {plane }}$ on the given region. Because only the compact phase and the plane-stress phase compete in this region, we conclude that the plane-stress phases $G$ that produce the normalized energy $2 \beta^{-1} \Psi_{\alpha \beta}^{\text {plane }}:=2 r+1+r^{-1}$ correspond to stable disarrangement phases for simple shear on the present region.

- For the region $\lambda>1,1<r<\lambda^{1 / 2}$, the argument just given shows that $\Psi_{\alpha \beta}^{\text {comp }}>\Psi_{\alpha \beta}^{\text {plane }}$ on the present region, as well, because the inequalities $1<r<\lambda^{1 / 2}$ imply the inequalities $1<r<\lambda$ employed in the previous region. The comparision of $2 \beta^{-1} \Psi_{\alpha \beta}^{u n i a x}:=3 r+r^{-2}$ and $2 \beta^{-1} \Psi_{\alpha \beta}^{\text {plane }}=$ $2 r+1+r^{-1}$ amounts to comparing $r+r^{-2}$ and $1+r^{-1}$, and it is easy to show that, for $r>1, r+r^{-2}>1+r^{-1}$, so that $\Psi_{\alpha \beta}^{\text {uniax }}>\Psi_{\alpha \beta}^{\text {plane }}$ on the 
present region. (Actually, an elementary argument allows us to conclude that

$$
\Psi_{\alpha \beta}^{\text {comp }}>\Psi_{\alpha \beta}^{\text {uniax }}>\Psi_{\alpha \beta}^{\text {plane }}
$$

on this region.) Therefore, the plane-stress disarrangement phases $G$ that produce the normalized energy $2 \beta^{-1} \Psi_{\alpha \beta}^{\text {plane }}:=2 r+1+r^{-1}$ also corresponds to a stable disarrangement phase for simple shear on the region $\lambda>1,1<$ $r<\lambda^{1 / 2}$.

- For $\lambda>1,0<r<1$ we repeatedly use the arithmetic-geometric mean inequality

$$
p_{1}+\cdots+p_{k} \geq k\left(p_{1} \cdots p_{k}\right)^{1 / k}
$$

for various values of the positive integer $k$ and the positive numbers $p_{1}, \ldots, p_{k}$ to conclude that

$$
\begin{aligned}
r+1+\lambda+\lambda^{-1} & \geq 2 r^{1 / 2}+2 \\
& =r^{1 / 2}+r^{1 / 2}+1+1 \geq 3 r^{1 / 3}+1 \\
& =r^{1 / 3}+r^{1 / 3}+r^{1 / 3}+1 \geq 4 r^{1 / 4}
\end{aligned}
$$

and, therefore, that on $\lambda>1,0<r<1$ there holds

$$
\Psi_{\alpha \beta}^{\text {comp }}>\Psi_{\alpha \beta}^{\text {plane }}>\Psi_{\alpha \beta}^{\text {uniax }}>\Psi_{\alpha \beta}^{\text {loose }}
$$

Consequently, loose disarrangement phases provide stable disarrangement phases in simple shear in this region.

The arguments above show that, for simple shear, in the only region $\lambda>$ $1,0<r<1$ where loose disarrangement phases can compete, they are necessarily stable, in agreement with the general conclusion drawn in Section 2. Our 
arguments also show that the compact phase competes in all cases, again in agreement with Section 2. However, for simple shear, we can conclude from above that the compact phase in every region provides the largest energy among the competing phases and that the only region where the compact phase is a stable disarrangement phase is the one in which it is the sole competitor for stability. The stable phases for simple shear that we have determined above as well as their regions of stability are depicted in Figure 5. The tables below

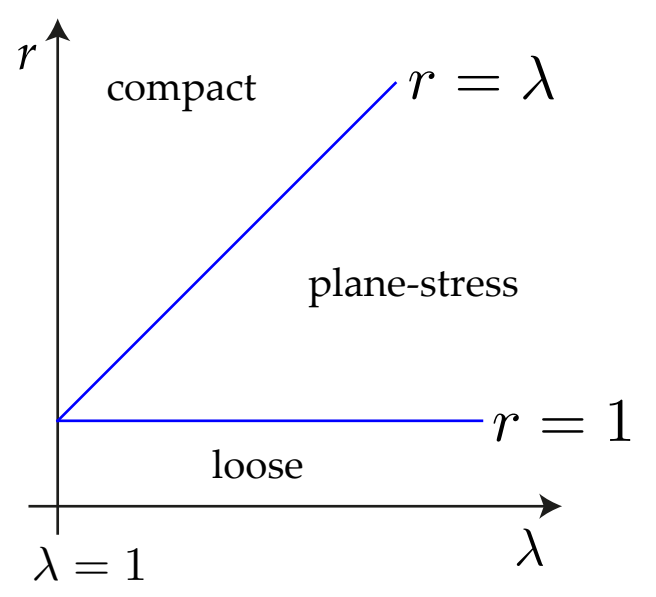

Figure 5: Stable phases for simple shear.

display these stable disarrangement phases for $F=I+\mu a \otimes b$, including information tabulated earlier for all disarrangement phases and refined here through our subsequent analysis. As in (57), we have $\lambda=1+\frac{\mu^{2}+|\mu|\left(\mu^{2}+4\right)^{1 / 2}}{2}$; moreover, the principal directions for $F$ are given by (58), and the principal directions for $G$ in the plane-stress category are restricted as indicated: 


\begin{tabular}{|c|c|c|}
\hline Category & Stable phase(s) $G$ & Principal directions of $G$ \\
\hline Compact & $F$ & $e_{i}^{F}, i=1,2,3$ \\
\hline Plane-stress & $(75)$ & $e= \pm e_{2}^{F}, f=\cos \theta e_{1}^{F}+\sin \theta e_{3}^{F}, \pm e_{2}^{F} \times f$ \\
\hline Loose & $r^{1 / 8} R$ & all directions \\
\hline
\end{tabular}

\begin{tabular}{|c|c|c|c|}
\hline Category & Stability region & Energy $2 \beta^{-1} \Psi_{\alpha \beta}$ & Stress $\beta^{-1} T$ \\
\hline Compact & $1<\lambda, \lambda<r$ & $r+1+\lambda+\lambda^{-1}$ & $F F^{T}-r I$ \\
\hline Plane-stress & $1<\lambda, 1<r<\lambda$ & $2 r+1+r^{-1}$ & $(1-r) e \otimes e+\left(r^{-1}-r\right) f \otimes f$ \\
\hline Loose & $\lambda \geq 1,0<r<1$ & $4 r^{1 / 4}$ & 0 \\
\hline
\end{tabular}

The entry "Stable phase(s) G" for the plane-stress category is the following expression:

$$
\begin{aligned}
G= & r^{1 / 2}(e \times f) \otimes\left(\frac{F^{-1} e}{\left|F^{-1} e\right|} \times \frac{F^{-1} f}{\left|F^{-1} f\right|}\right)+ \\
& +e \otimes \frac{F^{-1} e}{\left|F^{-1} e\right|}+r^{-1 / 2} f \otimes \frac{F^{-1} f}{\left|F^{-1} f\right|}
\end{aligned}
$$

and, in the entry "Principal directions of $G$ " for the plane-stress category, the angle $\theta$ is restricted through the relation

$$
\cos ^{2} \theta=\frac{\lambda(\lambda-r)}{\lambda^{2}-1}
$$

The information in Figure 5 and in the tables provides a landscape of the stable disarrangement phases available in simple shear. In the compact phase, because $G=F$, the deformation without disarrangement $G$ that identifies the phase is completely specified once $F$ is given. In the loose phases, $G$ is the scalar $r^{1 / 8}$ multiplied by an arbitrary rotation $R$. In the plane-stress phase, , the stable disarrangement phase $G$ is completely determined by the four vectors $e, f, F^{-1} e$, and $F^{-1} f$, with $e$ and $f$ given in the table. Consequently, apart from a choice of 
sign for $e$ and choices of signs in solving the relations $\cos ^{2} \theta=\frac{\lambda(\lambda-r)}{\lambda^{2}-1}$ for $\cos \theta$ and $\sin ^{2} \theta=\frac{\lambda r-1}{\lambda^{2}-1}$ for $\sin \theta$, the stable plane-stress phase $G$ is completely determined by $F=I+\mu a \otimes b$. The rotation $R_{G}$ corresponding to this stable disarrangement phase is given in Remark 2, and the principal stretches of $B_{G}=G G^{T}$ are $\lambda_{1}^{G}=r$, $\lambda_{2}^{G}=1$, and $\lambda_{3}^{G}=r^{-1}$.

In the compact phase, the Cauchy stress $T$ has principal stresses $\beta(\lambda-r)$, $\beta(1-r), \beta\left(\lambda^{-1}-r\right)$, so that, in the region of stability for the compact phase, the principal stresses are all negative and vary with the stretch $\lambda$. In the stable, plane stress phase, the formula in the table gives the Cauchy stress $T$ as a linear combination of the dyads $e \otimes e$ and $f \otimes f$ in which only the dyad $f \otimes f$ varies with the stretch $\lambda$. The principal stresses for this stable disarrangement phase are $0, \beta(1-r)$ and $\beta\left(r^{-1}-r\right)$, the latter two of which are negative in the region of stability for this disarrangement phase and do not vary with $\lambda$. In the loose phase, the stress vanishes as do all the principal stresses. Thus, for all of the stable disarrangement phases, the non-zero principal stresses are negative in the region of stability. In the two regions where the compact phase is not a stable disarrangement phase, the stable phase is such that one principal stress vanishes or all three principal stresses vanish, and all of the principal stresses are independent of the stretch $\lambda$. We conclude that the requirement of stability for a disarrangement phase in the case of simple shear entails all of the pieces of the aggregate to be subject to no tensile tractions, i.e., $T n \cdot n \leq 0$ for all unit vectors $n$, and stability for the compact disarrangement phase is characterized by the condition: $T n \cdot n<0$ for all unit vectors $n$. Consequently, the stable disarrangement phases in simple shear for an aggregate with the energy $\Psi_{\alpha \beta}$ display the characteristics of a "no-tension or masonry-like material with nonlinear elastic response" (see [4], [5], [6] for studies of no-tension/masonry-like materials with linear elastic response). 


\subsection{Shapes and orientations of deformed pieces of the ag- gregate in stable phases for simple shear}

We recall that for every structured deformation, the Approximation Theorem permits us to identify the deformation without disarrangements $G$ as the contribution at the macrolevel of submacroscopic deformations without disarrangements. A form of this identification relation that is useful here is the formula (see [1]):

$$
G(X)=\lim _{\varepsilon \longrightarrow 0} \lim _{n \longrightarrow \infty} \frac{\int_{B(X, \varepsilon)} \nabla f_{n}(Y) d V_{Y}}{\operatorname{vol} B(X, \varepsilon)}
$$

We can think of the size of the pieces of the aggregate going to zero as the index $n$ tends to infinity, so that, in the context of aggregates, $G(X)$ represents the average deformation $\frac{\int_{B(X, \varepsilon)} \nabla f_{n}(Y) d V_{Y}}{\operatorname{vol} B(X, \varepsilon)}$ of pieces of the aggregate within a ball of radius $\varepsilon$, computed in the limit as the size of the pieces tends to zero and, subsequently, as $\varepsilon$ tends to zero.

We describe here the geometry of the deformed pieces of the aggregate in terms of the principal stretches of $G$ for each stable disarrangement phase in simple shear. The following table displays these principal stretches along with their effect on a unit sphere:

\begin{tabular}{|c|c|c|}
\hline Stable phase & Principal stretches of $G$ & Image of unit sphere under $G$ \\
\hline Compact & $\lambda^{1 / 2}, 1, \lambda^{-1 / 2}$ & ellipsoid; shape depends on $\lambda$ \\
\hline Plane-stress & $r^{1 / 2}, 1, r^{-1 / 2}$ & ellipsoid; shape independent of $\lambda$ \\
\hline Loose & $r^{1 / 8}, r^{1 / 8}, r^{1 / 8}$ & sphere; size independent of $\lambda$ \\
\hline
\end{tabular}

The table shows that the only stable phase in which the shape of deformed pieces of the aggregate depends upon the macroshear $\lambda$ is the compact phase. Of course, in this phase $G=F$, and we cannot distinguish the geometrical changes in the pieces of the aggregate from those in the aggregate, itself. In 
the loose phases of the aggregate, the deformation without disarrangement $G$ causes changes in the pieces of the aggregate that, on average, take the unit sphere into a sphere of radius $r^{1 / 8}$, independent of the macroshear $\lambda$. All vectors are principal directions of $G$ for these loose phases, so that the principal directions also are independent of $\lambda$. By contrast, in each stable, plane-stress phase the deformation of the pieces of the aggregate, on average, takes the unit sphere into an ellipsoid with semiaxes of length $r^{1 / 2}, 1, r^{-1 / 2}$. The amount of macroshear $\lambda$ of the aggregate does not influence the deformed shape of the pieces.

From the results obtained in the appendix of [22], we must keep in mind that the principal directions $f$ and $e \times f$ of $G$ in the stable plane-stress phase do depend in a non-trivial way on the macroshear through the relations

$$
\begin{aligned}
& e_{3}^{G}=f=\cos \theta e_{1}^{F}+\sin \theta e_{3}^{F}, \text { with } \theta \text { satisfying (76) } \\
& e_{1}^{G}= \pm e_{2}^{F} \times f= \pm\left(\sin \theta e_{1}^{F}-\cos \theta e_{3}^{F}\right)
\end{aligned}
$$

When $\lambda=r$ the relation (76) yields $\cos ^{2} \theta=0$, and we obtain

$$
\begin{array}{ll}
e_{3}^{G} & \mid \lambda=r= \pm e_{3}^{F} \\
e_{1}^{G} & \backslash \quad \lambda=r= \pm e_{1}^{F}
\end{array}
$$

and we conclude that the principal directions of $F$ and of $G$ coincide when $\lambda=r$. As $\lambda \longmapsto \infty$ the relation (76) yields $\cos ^{2} \theta \longrightarrow 1$, and we obtain

$$
\begin{aligned}
& e_{3}^{G} \mid \lambda \longrightarrow \infty= \pm e_{1}^{F} \\
& e_{1}^{G} \mid \lambda \longrightarrow \infty=\mp e_{3}^{F}
\end{aligned}
$$

and this tells us that, in the limit as the stretch $\lambda$ tends to $\infty$, the principal 
directions of $G$ in the plane of shearing are again principal directions of $F$, but corresponding to different amounts of stretch. These results tell us that the orientations of the deformed pieces of the aggregate as they appear in the stable, plane-stress phase when $\lambda=r$ are the same as the orientation of the principal directions of the simple shear $F$. For values of stretch $\lambda$ greater than $r$ the orientation of the deformed pieces in the plane of shearing is rotated with respect to the principal directions of $F$ by an angle that tends to $\pm \pi / 2$ as the stretch increases without bound. In otherwords, while the shapes of the pieces of the aggregate do not vary with the macrostretch $\lambda$, their orientations relative to the principal directions of $F$ in the stable plane-stress phase for large macroshears $\lambda$ are rotated in the plane of shearing by an amount that tends to $\pm \pi / 2$ (see Figure 6).
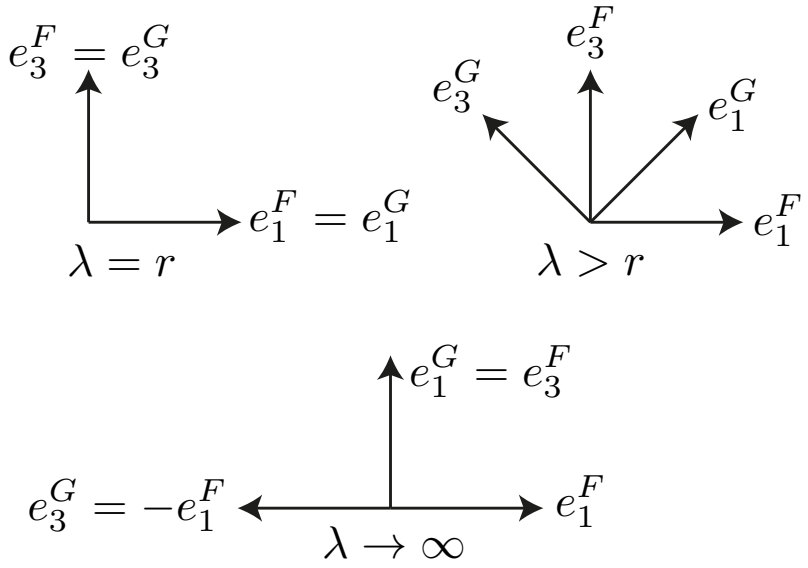

Figure 6: Relative orientation of principal axis of $G$ and $F$. 


\section{References}

[1] Del Piero, G., Owen, D.R.: Structured deformations of continua. Arch. Rat. Mech. Anal. 124 99-155 (1993)

[2] Deseri, L., Owen, D.R.: Toward a field theory for elastic bodies undergoing disarrangements. J. Elas. 70 197-236 (2003)

[3] Deseri, L., Owen, D.R.: Submacroscopically stable equilibria of elastic bodies undergoing dissipation and disarrangements. Math. Mech. Solids 15 611$638(2010)$

[4] Del Piero, G.: Limit analysis and no-tension materials. Int. J. Plasticity 14 259-271 (1998)

[5] Angelilo, M.: Constitutive relations for no-tension materials. Meccanica 28 195-202 (1993)

[6] Lucchesi, M., Padovani, C., Pagni, A.: A numerical method for solving equilibrium problems of masonry-like solids. Meccanica 29 175-193 (1994)

[7] Deseri, L., Owen, D.R.: Moving interfaces that separate loose and compact phases of elastic aggregates: a mechanism for drastic reduction or increase in macroscopic deformation. Continuum Mech. Thermodyn. 25 311-341 (2013) DOI:10.1007/s00161-012-0260-y

[8] Khakhar, D.: Rheology and mixing of granular materials. Macromol. Mater. Eng. 296 278-289 (2011)

[9] Alam, M., Luding, S.: First normal stress difference and crystallization in a dense sheared granular fluid. Phys. Fluids 15 2298-2312 (2003)

[10] Mueggenburg, N.: Behavior of granular materials under cyclic shear. Phys. Rev. E 031301-0313010 (2005) 
[11] Del Piero, G., Owen, D. R.: Integral-gradient formulae for structured deformations, Arch. Rat. Mech. Anal. 131 121-138 (1995)

[12] Del Piero, G., Owen, D. R.: Structured Deformations. XXII Scuola Estiva di Fisica Matematica, Ravello - Settembre 1997, Quaderni dell' Istituto Nazionale di Alta Matematica, Gruppo Nazionale di Fisica Matematica $(2000)$

[13] Dal Corso, F., Bigoni, D.: Growth of slip surfaces and line inclusions along shear bands in a softening material. Int. J. Fracture 166 225-237 (2010)

[14] Bigoni, D.: Nonlinear Solid Mechanics: Bifurcation Theory and Material Instability, Cambridge University Press (2012)

[15] Dal Corso, F., Deseri, L.: Residual stresses in random elastic composites: nonlocal micromechanics-based models and first estimates of the representative volume element size. DOI: 10.1007/s11012-013-9713-z Meccanica (2013)

[16] Lopez-Pamies, O., Ponte Castaẽda, P.: Second-Order Estimates for the Macroscopic Response and Loss of Ellipticity in Porous Rubbers at Large Deformations. J. Mech. Phys. Solids 76 (3) 247-287 (2004)

[17] Piccolroaz, A., Bigoni, D., J.R., Willis: A dynamical interpretation of flutter instability in a continuous medium. J. Mech. Phys. Solids 54 2391-2417 $(2006)$

[18] Oda, M., Iwashita, K.: Mechanics of Granular Materials: An Introduction, Taylor and Francis (1999)

[19] Ciarlet, P.G.: Mathematical elasticity, vol. I: Three Dimensional Elasticity. Studies in Mathematics and its Applications. vol. 20, North Holland, Amsterdam (1988) 
[20] Deseri, L., Owen, D. R.: Stable Disarrangement Phases of Granular Media, Parts I and II, Center for Nonlinear Analysis Research Reports Numbers 14-CNA-005, 14-CNA-006 (2014)

[21] Deseri, L., Owen, D. R.: Stable Disarrangement Phases of Elastic Aggregates: a Setting for the Emergence of No-tension Materials with Non-linear Response in Compression. Meccanica 49 2907-2932 (2014) doi::10.1007/s11012-014-0042-7

[22] Deseri, L., Owen, D. R.: Stable Disarrangement Phases Arising from Expansion/Contraction or from Simple Shearing of a Model Granular Medium, Center for Nonlinear Analysis Research Reports Number 14CNA-030 (2014)

[23] Deseri, L., Owen, D. R.: Submacroscopic disarrangements induce a unique, additive and universal decomposition of continuum fluxes, J. Elasticity (to appear).

[24] Owen, D.R., Paroni, R.: Second-order structured deformations, Arch. Rational Mech. Analysis 155 215-235 (2000) 


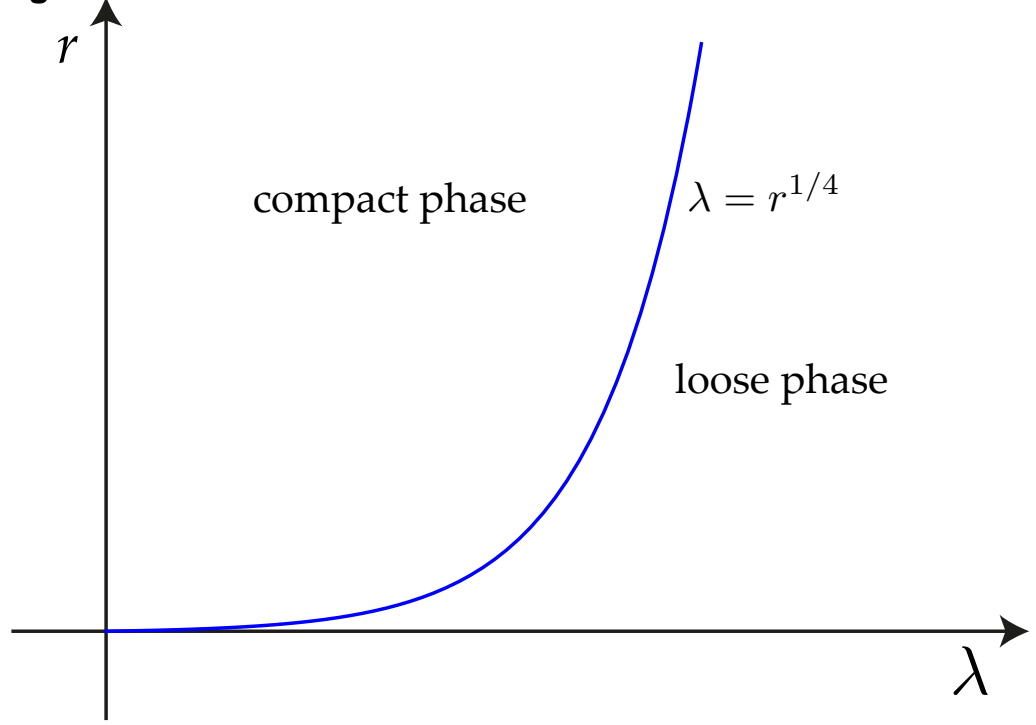


$\underset{r}{\text { Figure } 3}$

$r \uparrow$

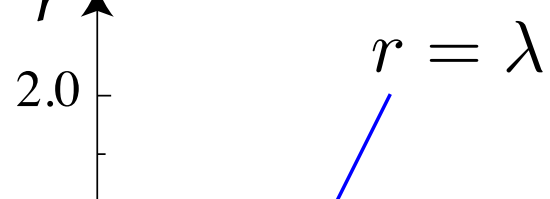

$2 r+1+r^{-1}$

1.0

0.5

$2+2 r^{1 / 2}$

$2 r+1+r^{-1}$

$r=1$



0.0

$1, \ldots, 2$

$2+1+1$

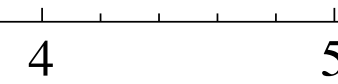

5 


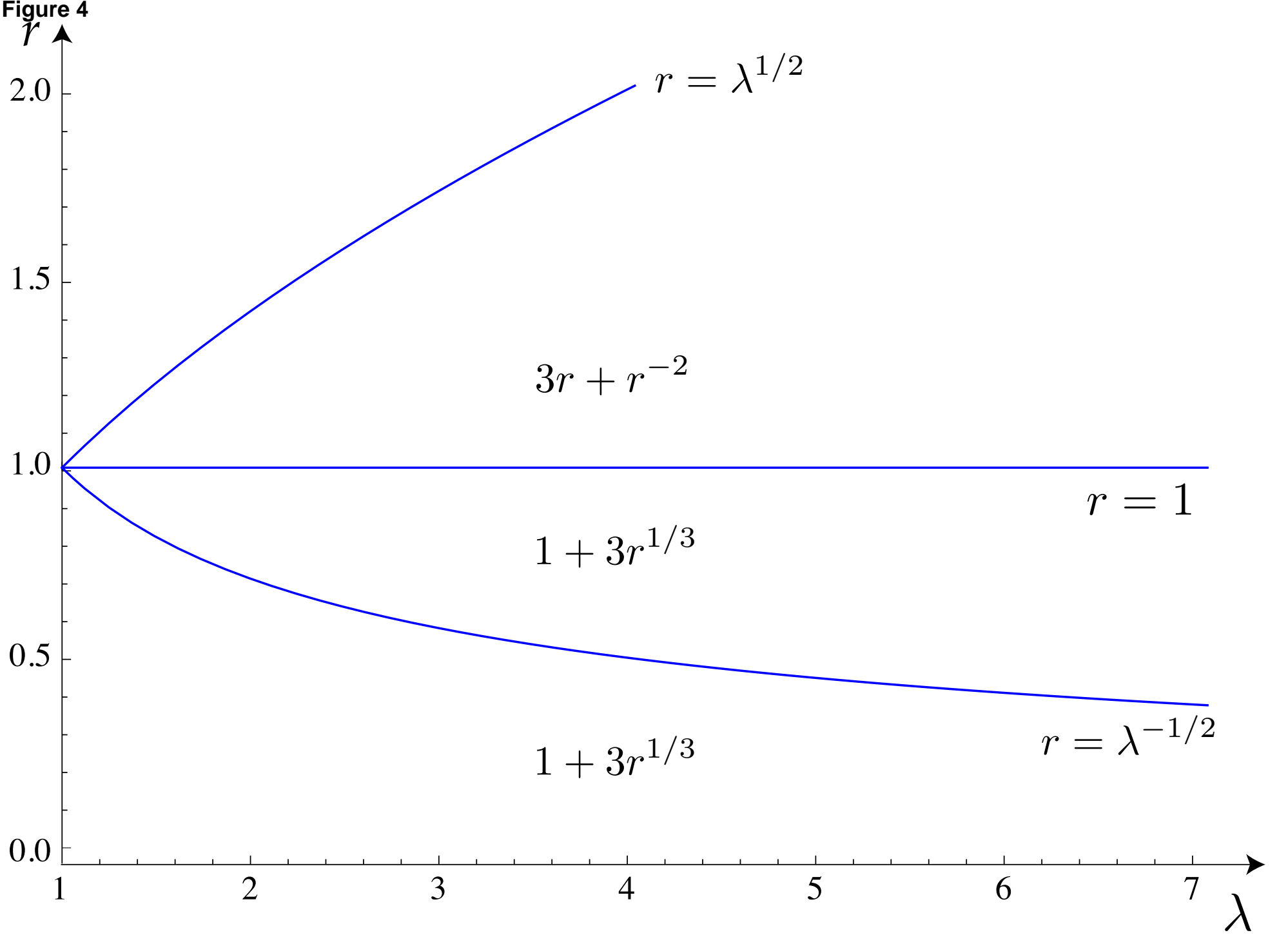


Figure 5

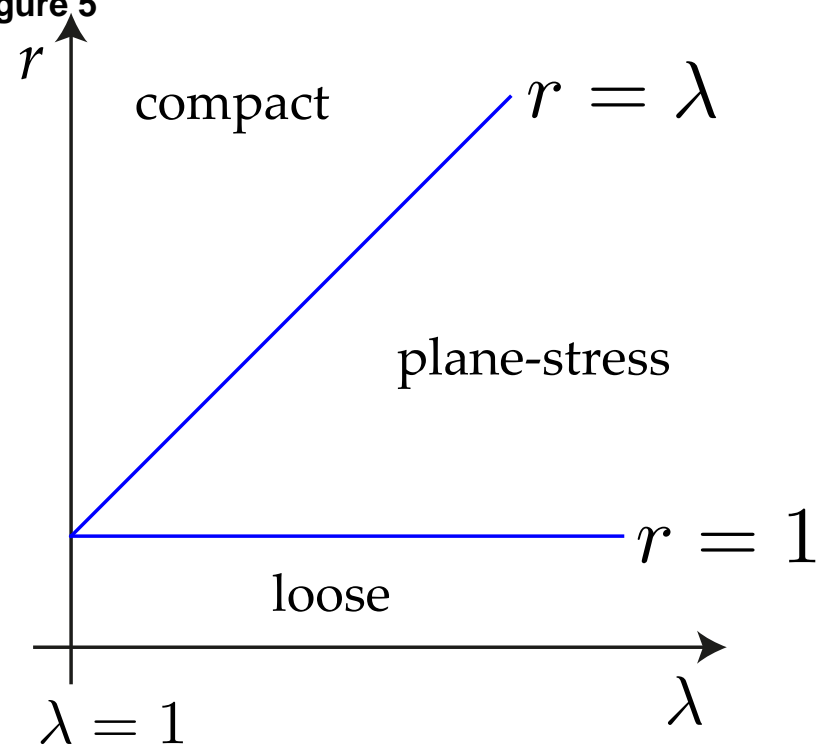


Figure 6

$e_{3}^{F}=e_{3}^{G}$

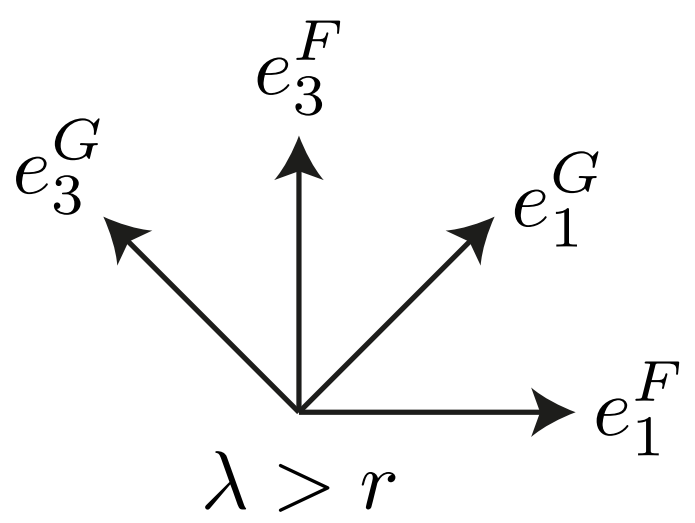

$e_{3}^{G}=-e_{1}^{F} \stackrel{\uparrow e_{1}^{G}=e_{3}^{F}}{\lambda \rightarrow \infty} \longrightarrow e_{1}^{F}$ 